\title{
THE DYNAMICS OF ROTATING WAVES IN SCALAR REACTION DIFFUSION EQUATIONS
}

\author{
S. B. ANGENENT AND B. FIEDLER
}

\begin{abstract}
The maximal compact attractor for the RDE $u_{t}=u_{x x}+f\left(u, u_{x}\right)$ with periodic boundary conditions is studied. It is shown that any $\omega$-limit set contains a rotating wave, i.e., a solution of the form $U(x-c t)$. A number of heteroclinic orbits from one rotating wave to another are constructed. Our main tool is the Nickel-Matano-Henry zero number. The heteroclinic orbits are obtained via a shooting argument, which relies on a generalized Borsuk-Ulam theorem.
\end{abstract}

1. Introduction. We investigate the global dynamics of the scalar reaction diffusion equation (RDE) on the circle

$$
u_{t}=u_{x x}+f\left(u, u_{x}\right), \quad x \in S^{1}=\mathbb{R} / 2 \pi \mathbb{Z},
$$

with periodic boundary conditions for $x \in[0,2 \pi]$, and under suitable assumptions on $f$. Equation (1.1) admits special solutions of the form $u(t, x)=U(x-c t)$ which are called rotating waves. Using zero-number (or lap-number)-type arguments we obtain the following two main results. The $\omega$-limit set of any trajectory of (1.1) contains a rotating wave or a steady state (Theorem 3.1). Any rotating wave or steady state $U$ with unstable dimension $i(U)$ connects to at least $[i(U) / 2]+2$ other rotating waves or steady states (Theorems $4.1,4.2$ ). These results can be seen as an attempt to describe the dynamics of (1.1) on the maximal compact attractor.

We assume throughout that the nonlinearity $f(u, p)$ satisfies

(1.2a) $f: \mathbf{R}^{2} \rightarrow \mathbb{R}$ is real analytic.

(1.2b) There exists $K_{1}>0$ such that $u f(u, 0)<0$ for all $u$ with $|u| \geq K_{1}$. By the first assumption, the initial-value problem associated to (1.1) defines a local semiflow $\phi_{t}$ on the Sobolev space $X=H^{s}\left(S^{1}\right)$ :

$$
\phi_{t}: X \rightarrow X \quad(0 \leq t), \quad u_{0} \mapsto \phi_{t}\left(u_{0}\right):=u(t, \cdot),
$$

where $u(t, x)$ denotes the maximal solution of $(1.1)$ with $u(0, \cdot)=u_{0}$. We pick $s>3 / 2$ so that $X$ embeds into $C^{1}\left(S^{1}\right)$.

Since our nonlinearity is real analytic, solutions of (1.1) are real analytic functions for $t>0$ and $x \in S^{\mathbf{1}}$ (see [29]).

Assumptions (1.2a) and (1.2b) are not strong enough to guarantee the existence of a global semiflow $\phi_{t}$ on $X$. In order to obtain a global semiflow we supplement the conditions $(1.2 \mathrm{a}, \mathrm{b})$ by assuming that the nonlinearity has at most subquadratic growth in $p$. More precisely we assume that there exist constants $C>0,0<\gamma<2$,

Received by the editors May 13, 1986 and, in revised form, April 18, 1987.

1980 Mathematics Subject Classification (1985 Revision). Primary 35K57; Secondary 58F 12.

This work was supported by the Deutsche Forschungsgemeinschaft. 
such that

$$
|f(u, p)| \leq C\left(1+|p|^{\gamma}\right)
$$

uniformly for $u$ in compact intervals.

Global existence of solutions $u(t, x)$ is known, even if $f(u, p)$ grows quadratically in $p$ [2]. The reader may also wish to consult [41, pp. 110, 111] for an example of blow-up in finite time when $f(u, p)$ grows superquadratically in $p$. We assume subquadratic growth in order to ensure existence of $\omega$-limit sets and of the maximal compact attractor $A$ (cf. [2, Theorem 5.3]). These sets are defined as follows. For any trajectory $\left\{\phi_{t}\left(u_{0}\right): t \geq 0\right\}$ the $\omega$-limit set is given by

$$
\omega\left(u_{0}\right)=\left\{u \in X: \exists t_{k} \uparrow \infty \text { such that } \phi_{t_{k}}\left(u_{0}\right) \rightarrow u\right\} .
$$

The maximal compact attractor of (1.1) is defined by

$$
\begin{aligned}
& A=\left\{u \in X: \exists t_{k} \uparrow \infty \text { and } u_{k} \in X\right. \text { such that } \\
& \left.\qquad \sup \left\|u_{k}\right\|<\infty \text { and } \phi_{t_{k}}\left(u_{k}\right) \rightarrow u\right\} .
\end{aligned}
$$

For any $u_{0} \in A$ the trajectory $\left\{\phi_{t}\left(u_{0}\right)\right\}_{t \geq 0}$ may be uniquely extended to negative $t$ (backward uniqueness holds here since all orbits are real analytic).

We refer the reader to Henry's book [20] for more information on general semiflows like $\left\{\phi_{t}\right\}$, and to [19] for a survey on maximal compact attractors.

This paper is devoted to a qualitative description of both $\omega\left(u_{0}\right)$ and $A$, much in the spirit of Hale [19]. Note that $A$ consists of all $\omega$-limit sets, and their connecting orbits, which are defined as follows. Given nonempty subsets $A, B$ of $X$ we say that $A$ connects to $B$ if there exists a global trajectory $u(t, \cdot)=\phi_{t}\left(u_{0}\right), t \in \mathbb{R}$, such that

$$
A \subseteq \alpha\left(u_{0}\right), \quad B \subseteq \omega\left(u_{0}\right),
$$

where the $\alpha$-limit set $\alpha\left(u_{0}\right)$ is defined like $\omega\left(u_{0}\right)$ but $t_{k} \rightarrow \infty$ is replaced by $t_{k} \rightarrow$ $-\infty$. We call $u(t, \cdot)$ a connecting orbit or an (orbit) connection.

A principal feature of equation (1.1), which is reflected in $\omega$ and $A$, is equivariance with respect to the group $S^{1}$ acting by shifting $x$. Indeed let

$$
R_{\theta}: X \rightarrow X, \quad\left(R_{\theta} u_{0}\right)(x):=u_{0}(x+\theta)
$$

denote the shift in $x$ by $\theta \in S^{1}=\mathbb{R} / 2 \pi \mathbb{Z}$. Then $\phi_{t}$ commutes with $R_{\theta}$,

$$
\phi_{t} \circ R_{\theta}=R_{\theta} \circ \phi_{t}
$$

because the nonlinearity $f$ does not depend on $x$. This motivates the following terminology. A steady state $U \in X$ is a solution of $(1.1)$ with $U_{t}=0$. We call $U$ homogeneous if $R_{\theta} U=U$ for all $\theta \in S^{1}$. Otherwise we call $U$ nonhomogeneous or standing wave. On the other hand, we call $U \in X$ a rotating wave of speed $c \neq 0$ if

$$
\left(\phi_{t} U\right)(x)=U(x-c t)=\left(R_{-c t} U\right)(x)
$$

i.e.

$$
-c U^{\prime}=U^{\prime \prime}+f\left(U, U^{\prime}\right) .
$$

For $c=0$, a rotating wave becomes a standing wave. Note that standing waves occur in circles given by $\left\{R_{\theta} U: \theta \in S^{1}\right\}$ (similarly, $A$ is invariant under the action $\left.R_{\theta}\right)$. By Corollary 3.2 any nonstationary time periodic orbit $u(t, \cdot)$ of $(1.1)$ is a 
rotating wave. But rotating waves are of a transient character: by Corollary 2.4 below they are all unstable. This is a manifestation of a more general phenomenon in order preserving systems; see $[23 ; 24$, Theorem $2.4 ; 37$, Theorem 2$]$ for a discussion. If $U$ is a hyperbolic rotating wave, i.e. a hyperbolic periodic orbit of (1.1), then its unstable manifold $W^{\mathrm{u}}(U)$ belongs to the maximal compact attractor $A$. The asymptotic behavior of the flow on $W^{\mathrm{u}}(U)$ seems to be governed by the dimension $i(U)$ of the (local) unstable manifold $W^{\mathrm{u}}$; we call $i(U)$ the instability index of $U$. For those who contend that rotating waves may not exist, we give an example below.

The main tool in our analysis of $\omega$-limit sets and the maximal compact attractor is the zero number. For any continuous $\phi: S^{1} \rightarrow \mathbb{R}$, the zero number $z(\phi)$ is the number of sign changes of $\phi$, not counting multiplicity; i.e. $z(\phi)$ is the maximal integer $n \leq \infty$ such that there exist $0 \leq x_{n+1}=x_{0}<x_{1}<\cdots<x_{n}<2 \pi$ with

$$
\phi\left(x_{i}\right) \cdot \phi\left(x_{i+1}\right)<0 \quad(0 \leq i \leq n) ;
$$

put $z(0):=0$. Note that $z$ is finite on $\mathcal{A}$, by analyticity, and is even. The crucial property of $z(\phi)$, going back to Nickel [40] essentially and revived by Matano [35] and Henry [21], is the following. For any solution $v(t, x)$ of the $x$-dependent equation

$$
v_{t}=v_{x x}+g\left(x, v, v_{x}\right), \quad x \in S^{1}
$$

with $g$ sufficiently regular and $g(x, 0,0)=0$ the function $t \rightarrow z(v(t, \cdot))$ is nonincreasing with $t$. The proof just uses maximum principles; see e.g. [35, 7]. Analyticity is used below, to obtain a sharper version of this result: $z(v(t, \cdot))$ drops if, and only if, $x \rightarrow v(t, x)$ has a multiple zero for some $x$ (Corollary 5.3).

We can now formulate our main results. Theorem 3.1 states that any $\omega$-limit set $\omega\left(u_{0}\right)$ has to contain a rotating wave or a steady state. In contrast, for the scalar equation

$$
u_{t}=u_{x x}+f(u)
$$

$x \in[0,1]$ with various boundary conditions, it is known that $\omega\left(u_{0}\right)$ consists of exactly one stationary solution [35]. Only if all steady states and rotating waves are hyperbolic can we prove an analogous uniqueness statement for (1.1); see Theorem 3.3. Here we call a standing wave type steady state $U$ hyperbolic if $U_{x}$ (coming from the circle $R_{\theta} U$ of rotating waves) yields the only purely imaginary eigenvalue of the linearization, namely an eigenvalue 0 .

Concerning the rest of the maximal compact attractor $A$, i.e. orbit connections, we consider the unstable manifold $W^{\mathrm{u}}$ of a given steady state or rotating wave $U$ with instability index $i(U)$. Through it, $U$ connects to the closest homogeneous steady state above (resp. below) $U$ (Theorem 4.1 ), and to at least $[i(U) / 2]$ other rotating waves or steady states $w_{k}, 0<2 k \leq i(U)$ (Theorem 4.2). The zero number discerns them:

$$
z\left(w_{k}-U\right)=2 k .
$$

More generally, we may prescribe the graph of $t \rightarrow z(u(t, \cdot)-U)$ pretty much arbitrarily (but nonincreasing), and still find a trajectory $u(t, \cdot) \in W^{\mathrm{u}} \subseteq A$ which realizes this particular graph (Theorem 4.3).

These results belong to a series of attempts to understand the dynamics of some simple infinite-dimensional dynamical systems given by scalar equations. The global 
dynamics of (1.10) has been investigated e.g. in $[\mathbf{3}, \mathbf{7}, \mathbf{8}, \mathbf{1 1}, \mathbf{1 9 - 2 1}, \mathbf{3 5}, \mathbf{3 6}, \mathbf{4 3}$. One approach relies on Conley's index and the variational structure to establish connecting orbits; for a summary see $[43, \S \S 22-24]$. The variational structure of (1.10) comes in via the continuous Ljapunov functional

$$
\begin{aligned}
& V(u):=\int_{0}^{1}\left(\frac{1}{2} u_{x}^{2}-F(u)\right) d x, \quad F^{\prime}(u)=f(u), \\
& \frac{d}{d t} V(u(t, \cdot))=-\int_{0}^{1} u_{t}^{2} d x
\end{aligned}
$$

which forces $u(t, \cdot)$ to approach a steady state. The other approach relies on the discrete Ljapunov functional $z(u(t, \cdot))$; for a summary see [8]. While there are variational problems without any known $z$ (e.g. (1.10) with $x \in \Omega \subset \mathbb{R}^{n}, n \geq 2$ and $\Delta u$ instead of $u_{x x}$ ) there are also nonvariational problems which admit a discrete Ljapunov functional, e.g. (1.1) or differential-delay equations

$$
\dot{u}(t)=-f(u(t), u(t-1))
$$

with negative feedback $[\mathbf{3 1}, \mathbf{3 2}]$. Actually, $[\mathbf{3 1}, \mathbf{3 3}]$ contain results which are somewhat analogous to those presented here; Conley's index is used in [31] but not in [33]. A common feature, and indeed difficulty, of (1.1) and (1.11) is the occurrence of conjugate complex (nonreal) eigenvalues for the linearized equations. This led us to consider (1.1) with periodic boundary conditions. Besides, $\omega\left(u_{0}\right)$ would be just a steady state for $x \in[0,1]$ with say Dirichlet boundary conditions. In this context we note that Matano has constructed a Ljapunov functional of (1.1) with Dirichlet conditions [38].

To establish connections for (1.1), we follow the same basic idea as in [8]. We encode the dropping times in a mapping between spheres, which we call the $y$-map. Surjectivity of the $y$-map will provide lots of connections in Theorem 4.3. To prove $y$ is surjective, we actually prove $y$ is essential (i.e. not homotopic to a constant map) by homotopy to the case of linear $f$. For (1.10), i.e. with real eigenvalues, we invoke the Borsuk-Ulam theorem to prove $y$ is essential. This time we need the complex analogue of the Borsuk-Ulam theorem, due to Hopf and Rueff [26], because complex eigenvalues occur. Equivariance of (1.1) with respect to the action (1.7) of $R_{\theta}$ helps. Here is a concrete example for equation (1.1). Consider

$$
u_{t}=\varepsilon u_{x x}+g(u)_{x}+h(u), \quad x \in S^{1}=\mathbb{R} / 2 \pi \mathbb{Z}
$$

for small $\varepsilon>0$. To be specific we choose

$$
g(u)=\frac{1}{3} u^{3}, \quad h(u)=u\left(1-(\delta u)^{2}\right)
$$

with $\delta>0$ small. Rotating waves $U$ of (1.12) with speed $c$ are periodic solutions of

$$
\varepsilon U_{x x}+(g(U)+c U)_{x}+h(U)=0
$$

with minimal period $p>0$ such that $2 \pi=m p$ for some integer $m$. Writing $g_{c}(u):=g(u)+c u$, this is equivalent to the first-order system

$$
\varepsilon U_{x}=V-g_{c}(U), \quad V_{x}=-h(U) .
$$

For $\delta$ tending to zero, $h(u)=u+o(1)$ holds uniformly on compact subsets. For $h(u)=u$ and $\varepsilon$ small, (1.13) is the singularly perturbed Van der Pol oscillator; see 
$[17,28,39]$ for a detailed discussion. We introduced $\delta$ small to meet assumptions (1.2a), (1.2b). The reader can easily verify that (1.2c) is also satisfied. The periodic solultion of the singular Van der Pol oscillator is well known: it is a relaxation oscillation following $V=g_{c}(U)$ most of the time, except for two rapid transition layers of width $O\left(\varepsilon^{2 / 3}\right)$. The minimal period $p_{\varepsilon}$ is

$$
p_{\varepsilon}=(3-2 \log 2)|c|+O\left(\varepsilon^{2 / 3}\right)
$$

and the amplitude is approximately $2|c|^{3 / 2}$. These solutions exist only for $c<0$ : there are no rotating waves with $c \geq 0$. For the original system (1.12) we thus obtain a finite number of rotating waves $U^{m}$ with speeds

$$
c^{m}=-\frac{2 \pi}{3-2 \log 2} \cdot \frac{1}{m}+o(1),
$$

one for each $m$ with $1 \leq m \leq M_{\varepsilon}$, where $M_{\varepsilon} \rightarrow \infty$ for $\varepsilon \rightarrow 0$. The wave $U^{m}$ has $2 m$ rapid transition layers, $z\left(U^{m}\right)=2 m, i\left(U^{m}\right)=2 m-1$, and $U^{m}$ connects to each wave $U^{j}$ with $j<m$ (because the $U^{m}$ provide all rotating waves by uniqueness of the limit cycle of (1.13)), and to the two homogeneous steady states $U \equiv \pm 1 / \delta$. Moreover each $\omega$-limit set consists of exactly one of these waves or a homogeneous steady state.

Pursuing the viscosity limit $\varepsilon \rightarrow 0$ in (1.12), our rotating waves $U^{m}$ provide time-periodic solultions of the inhomogeneous scalar hyperbolic equation

$$
u_{t}-g(u)_{x}=h(u), \quad x \in S^{1}
$$

with $2 m$ admissible rotating shocks. We believe that our results on connecting orbits still hold for this case mutatis mutandis. In particular, the zero number $z(u(t, \cdot))$ should be nonincreasing along entropy solutions $u(t, \cdot)$ of $(1.14)$. Using characteristics, it is clear that $t \rightarrow z(u(t, \cdot))$ can change only at shocks. From the applications point of view, the homogeneous conservation law $h \equiv 0$ can be interpreted as a caricature of gas dynamics. In this case $u(t, \cdot)$ tends to equilibrium under suitable assumptions on $g[\mathbf{9}, \mathbf{1 3}, \mathbf{3 0}]$, and also $[43, \S 16]$. Similarly, equations (1.12) and (1.14) may be understood as a caricature of the dynamics of a reactive gas. However, we do not pursue the viscosity limit any further in this paper.

We did not try to keep this paper self-contained. We introduce the main concepts briefly, e.g. zero number, invariant manifolds, strong and fast (un)stable manifolds, normal hyperbolicity. For background information on zero number and invariant manifolds see [7]; for normal hyperbolicity see $[4,16,20,25]$. In [8] a rather selfcontained treatment of the simpler case (1.10), where $f$ is independent of $u_{x}$, has been given.

Our paper is organized as follows. In $\S 2$ we discuss the implications of $z$ for linear equations (Theorems 2.1 and 2.2). As a corollary we obtain that all rotating waves are unstable. $\S 3$ is devoted to our study of $\omega$-limit sets (Theorems 3.1 and 3.3) establishing the significance of rotating waves. Some connections from rotating and standing waves are discovered in $\S 4$ (Theorems 4.1 and 4.2) together with an analysis of $z(u(t, \cdot))$ on their unstable manifold (Theorem 4.3). We conclude the paper in $\S 5$ with an appendix on the dropping of $z(u(t, \cdot))$ for multiple zeros of $x \mapsto u(t, x)$ (Theorem 5.1).

ACKnowledgment. We are indebted to H.-W. Henn and D. Puppe for their benevolent help with topology, and to G. Keller for the slick application of Birkhoff's 
recurrence theorem in the proof of Lemma 2.3. The manuscript was typed carefully and efficiently by E. Urmitzer.

2. Spectral theory of the linearized flow. The Fréchet derivative of the flow $\phi_{t}$ on $X$ is given by the following recipe:

$$
w=d \phi_{t}\left(u_{0}\right) \cdot v
$$

holds iff there is a solution $U(s, x)$ of

$$
U_{s}=U_{x x}+a(s, x) U_{x}+b(s, x) U, \quad x \in S^{1}, 0 \leq s \leq t,
$$

such that

$$
U(0, x)=v(x), \quad U(t, x)=w(x), \quad x \in S^{1} .
$$

Here the coefficients $a$ and $b$ are given by

$$
\begin{aligned}
& a(s, x)=f_{p}\left(u(s, x), u_{x}(s, x)\right), \\
& b(s, x)=f_{u}\left(u(s, x), u_{x}(s, x)\right)
\end{aligned}
$$

for $x \in S^{1}$ and $0 \leq s \leq t$.

In this section we study the linear operator $T=d \phi_{t}\left(u_{0}\right): v \rightarrow w$ defined by $(2.1)$ and (2.2) for arbitrary real analytic functions $a(s, x)$ and $b(s, x)$ on $S^{\mathbf{1}} \times[0, t]$. It follows from standard regularity theory that $T$ is a bounded compact operator in the Hilbert space $X$ (see [20]). The Riesz-Schauder theory of compact operators then tells us that the spectrum of $T$ is given by a sequence of eigenvalues of finite multiplicity, $\left\{\lambda_{j}\right\}_{j=0,1,2, \ldots}$. We assume that these $\lambda_{j}$ are ordered by $\left|\lambda_{j}\right| \geq\left|\lambda_{j+1}\right|$, and that they are repeated in the sequence $\left\{\lambda_{j}\right\}_{j=0,1, \ldots}$ according to their algebraic multiplicity.

In the special case that the coefficients $a(s, x)$ and $b(s, x)$ do not depend on $s \in[0, t]$ the eigenfunctions of $T$ coincide with those of the differential operator

$$
A=\left(\frac{d}{d x}\right)^{2}+a(x) \frac{d}{d x}+b(x) \quad\left(x \in S^{1}\right),
$$

where $a(s, x) \equiv a(x)$ and $b(s, x) \equiv b(x)$. Using ODE techniques one can obtain a complete description of the oscillation properties of the eigenfunctions of $A$ (the basic ideas probably go back to Sturm).

The point we wish to make in this section is that the main results of the Sturm oscillation theory (i.e. Theorems 2.1 and 2.2 below) also hold true in the general case when $a$ and $b$ are allowed to depend on time.

Our first result in this direction is

THEOREM 2.1. Let $\left\{\lambda_{j}\right\}_{j \geq 0}$ be the ordered sequence of eigenvalues of $T$ repeated with algebraic multiplicity. Then for all $j \geq 0$ we have $\left|\lambda_{2 j}\right|>\left|\lambda_{2 j+1}\right|$.

This theorem implies that $\left\{\lambda_{2 j-1}, \lambda_{2 j}\right\}$, for $j \geq 1$, is a spectral set for the operator $T$. Let $E_{2 j}$ denote the real generalized eigenspace of $\left\{\lambda_{2 j-1}, \lambda_{2 j}\right\}$ for $j \geq 1$, and let $E_{0}$ be the real eigenspace corresponding to the isolated eigenvalue $\lambda_{0}$. In particular $\operatorname{dim}_{\mathbb{R}} E_{0}=1, \operatorname{dim}_{\mathbf{R}} E_{2 j}=2(j \geq 1)$ by Theorem 2.1 . 
Our other result is

THEOREM 2.2. Any nonzero $u \in E_{2 j}$ has only simple zeros, and moreover $z(u)=2 j$.

The proof of these two theorems will be based on the following lemma. First we introduce some more notation.

For $j=0,1,2, \ldots$ define $r_{j}=\left|\lambda_{j}\right|$, and let $F_{j} \subset X$ be the generalized eigenspace of $T$ belonging to all those eigenvalues $\lambda$ which satisfy $|\lambda|=r_{j}$. Then $F_{j}$ is finite dimensional.

LEMMA 2.3. Any nonzero real $u \in F_{j}$ has only simple zeros.

ProOF. Let $T \mid F_{j}$ have the Jordan decomposition $T \mid F_{j}=S+N$, with $S N=N S$, $S$ semisimple and $N$ nilpotent. Let a real $u \in F_{j} \backslash\{0\}$ be given. Then we can consider the sequence of vectors $u_{k} \in F_{j}$ given by $u_{k}=T^{k}(u)(k \in \mathbb{Z})$, and the corresponding sequence of integers $z_{k}=z\left(u_{k}\right)$.

Since the operator $T$ is defined by solving the parabolic equation (2.1), the sequence of zero numbers $z_{k}$ is nonincreasing. Moreover, if $u=u_{0}$ has a multiple zero then $z_{1}$ is strictly less than $z_{0}$ by Theorem 5.1 . Therefore it is sufficient to show that $z_{k}$ does not depend on $k$.

In order to do this we study the asymptotic behavior of the $u_{k}$ as $k \rightarrow \pm \infty$. The binomial theorem states that

$$
T^{k} \cdot u=\sum_{l=0}^{m}\left(\begin{array}{l}
k \\
l
\end{array}\right) S^{k-l} \cdot N^{l} \cdot u
$$

for any $k \in \mathbb{Z}$, where $m$ is the largest integer for which $N^{m} \cdot u \neq 0$ (recall that $N$ is nilpotent so that such an $m$ exists). In particular we have

$$
u_{k}=S^{k-m}\left\{\frac{k^{m}}{m !} N^{m} u+O\left(k^{m-1}\right)\right\} \quad(k \rightarrow \pm \infty) .
$$

Therefore $v_{k}$ defined by $v_{k}=k^{-m} r_{j}^{-(k-m)} u_{k}$ satisfies

$$
v_{k}=\left(\frac{S}{r_{j}}\right)^{k-m} \cdot \frac{N^{m}}{m !} u+o(1) \quad(k \rightarrow \pm \infty) .
$$

By construction the matrix $S / r_{j}$ is simple, with all its eigenvalues on the unit circle. We claim that there exist sequences $k_{n}^{ \pm}$, tending to $+\infty$ or $-\infty$, respectively, for which

$$
\left(S / r_{j}\right)^{k_{n}^{ \pm}-m} \rightarrow \mathrm{id} \quad \text { as } n \rightarrow \infty .
$$

Indeed, let $S / r_{j}$ be represented by the diagonal matrix $\operatorname{diag}\left(s_{1}, \ldots, s_{N}\right)$, where each $s_{j}$ satisfies $\left|s_{j}\right|=1$. Then $S / r_{j}$ acts on the $N$-torus

$$
T^{N}=\left\{\left(z_{1}, \ldots, z_{N}\right) \in \mathbb{C}^{N}|| z_{j} \mid=1\right\}
$$

by multiplication: $\left(z_{1}, \ldots, z_{N}\right) \mapsto\left(s_{1} z_{1}, \ldots, s_{N} z_{N}\right)$. By the Birkhoff recurrence theorem [42], there exists a $u^{*} \in T^{N}$ and sequences $k_{n}^{ \pm} \rightarrow \pm \infty$ such that $\left(S / r_{j}\right)^{k_{n}^{ \pm}-m} u^{*} \rightarrow u^{*}$. Canceling each component of $u^{*}$ on both sides, this proves our claim. For a reference see also [12, Chapter 3.1, Lemma 1]. 
Using our asymptotic expansion for the $v_{k}$ we get

$$
v_{k_{n}}^{ \pm} \rightarrow N^{m} u / m ! \quad(n \rightarrow \infty) .
$$

The convergence takes place in the $X$-topology, so that we certainly have $C^{1}$ convergence. Hence, if $N^{m} u$ has only simple zeros, then so will $v_{k_{n}}^{ \pm}$for large $n$, and in fact $z\left(u_{k_{n}}^{ \pm}\right)=z\left(v_{k_{n}}^{ \pm}\right)=z_{k_{n}}^{ \pm}=z(u)$ will be independent of $n$, which implies that $u$ has simple zeros.

Thus the proof will be complete if we can show that $N^{m}(u)$ has only simple zeros. Again, we look at a sequence of vectors

$$
w_{k}=\left(T / r_{j}\right)^{k} \cdot N^{m} u=\left(S / r_{j}\right)^{k} \cdot N^{m} u .
$$

As before, $z\left(w_{k}\right)(k \in \mathbb{Z})$ is nonincreasing (since $\left.N \cdot N^{m} u=0\right)$. It follows that $z\left(w_{k}\right)$ is constant for $k$ large, and that for any large integer $k_{0}, w_{k_{0}}$ will only have simple zeros (Theorem 5.1). Then, using the sequence $k_{n}^{-} \rightarrow-\infty$ defined above we see that

$$
w_{k_{n}^{-}-m+k_{0}}=\left(S / r_{j}\right)^{k_{n}^{-}-m} w_{k_{0}} \rightarrow w_{k_{0}} \quad(n \rightarrow \infty)
$$

so that $z\left(w_{k_{n}^{-}-m+k_{0}}\right)=z\left(w_{k_{0}}\right)$ if $n$ is large enough. This means that the sequence $z\left(w_{k}\right)$ is constant and that none of the $w_{k}$ can have a multiple zero. Hence $N^{m} u=$ $w_{0}$ has only simple zeros, and the proof is complete.

PROOF OF THEOREMS 2.1 AND 2.2. If the coefficients $a(t, x), b(t, x)$ in $(2.1)$ vanish identically, then (2.1) becomes the standard heat equation and the eigenvalues $\lambda_{j}$ and the spaces $E_{2 j}$ defined above are given explicitly by

$$
\begin{gathered}
\lambda_{0}=0, \quad E_{0}=\{\xi \mid \xi \in \mathbb{R}\}, \\
\lambda_{2 j-1}=\lambda_{2 j}=e^{-j^{2} t}, \quad E_{2 j}=\left\{\xi_{j} \cos j x-\eta_{j} \sin j x \mid \xi_{j}, \eta_{j} \in \mathbb{R}\right\} .
\end{gathered}
$$

In particular we see that Theorems 2.1 and 2.2 are true in this case. We shall now present a continuation argument which shows that these theorems also hold for general $a$ and $b$.

For any $\theta \in[0,1]$ we consider an operator $T^{\theta}$ on $X$ defined by $w=T^{\theta} \cdot v$ iff there is a solution $U(s, x)$ of

$$
\begin{gathered}
U_{s}=U_{x x}+\theta a(s, x) U_{x}+\theta b(s, x) U, \quad x \in S^{1}, 0 \leq s \leq t, \\
U(0, x)=v(x), \quad U(t, x)=w(x) \quad\left(x \in S^{1}\right) .
\end{gathered}
$$

Then we have a one parameter family of eigenvalues $\lambda_{j}(\theta)$ of $T^{\theta}$. As before, we define $r_{j}(\theta)=\left|\lambda_{j}(\theta)\right|$, and we let $F_{j}(\theta)$ denote the generalized eigenspace of $T^{\theta}$ corresponding to those eigenvalues $\lambda$ with $|\lambda|=r_{j}(\theta)$.

Since $T^{\theta}: X \rightarrow X$ depends continuously on $\theta \in[0,1]$, perturbation theory $[\mathbf{2 7}$, §IV.3] implies that the $r_{j}(\theta)$ are continuous functions of $\theta$. The subspaces $F_{j}(\theta)$ vary upper semicontinuously with $\theta$ : given $u_{n} \in F_{j}\left(\theta_{n}\right),\left|u_{n}\right| \leq 1$, for $\theta_{n} \rightarrow \theta_{0}$, there exists a convergent subsequence of $u_{n}$ converging to $u_{0} \in F_{j}\left(\theta_{0}\right)$.

Now define the integer $z_{j}(\theta)$ to be the zero number of some $u \in F_{j}(\theta) \backslash\{0\}$. By Lemma 2.3, $z_{j}(\theta)$ is independent of the choice of $u$. We claim that $z_{j}(\theta)$ is continuous in $\theta$ (and hence independent of $\theta$ ). Indeed, if $\theta_{n} \rightarrow \theta_{0}$ we may choose $u_{n} \in F_{j}\left(\theta_{n}\right),\left|u_{n}\right|=1$ converging to $u_{0} \in F_{j}\left(\theta_{0}\right)$ in $X$ and hence in $C^{1}$. By 
Lemma 2.3 and $\left|u_{0}\right|=1$, all zeros of $u_{0}$ are simple. Therefore $z_{j}\left(\theta_{n}\right)=z_{j}\left(\theta_{0}\right)$ for $n$ sufficiently large.

Thus the $z_{j}(\theta)$ are constant. At $\theta=0$ one easily sees that

$$
z_{2 j-1}(\theta)=z_{2 j}(\theta)=2 j \quad(j=1,2, \ldots)
$$

and $z_{0}(\theta)=0$. This implies that $r_{2 j}(\theta)>r_{2 j+1}(\theta)$ for all $\theta$, as $r_{2 j}=r_{2 j+1}$ would force $z_{2 j}$ and $z_{2 j+1}$ to be equal. Theorem 2.1 is therefore true. To complete the proof of Theorem 2.2 we observe that Theorem 2.1 implies that the spaces $E_{2 j}$ and $F_{2 j}$ coincide, so that Theorem 2.2 follows from Lemma 2.3.

COROLlARY 2.4. Let $U$ be a (rotating or standing) wave. Then $i(U) \in\left\{z\left(U_{x}\right)\right.$ $\left.-1, z\left(U_{x}\right)\right\}$. In particular, all waves are unstable.

PrOOF. We give a proof in case $U$ is a standing wave. The case of a rotating wave is analogous by the transformation $x \mapsto x-c t$. Obviously, $U_{x} \not \equiv 0$ is an eigenfunction of $T$ with eigenvalue 1 . Thus $1 \in\left\{\lambda_{2 j-1}, \lambda_{2 j}\right\}$ with $2 j:=z\left(U_{x}\right)$ by Theorem 2.2 and $2 j-1 \leq i(U) \leq 2 j$ by Theorem 2.1. This proves the corollary.

3. The $\omega$-limit set. Let $u_{0} \in X$ be an arbitrary initial value. Then, as we noted in the introduction, there exists a global solution $u(t, \cdot)=\phi_{t}\left(u_{0}\right)(t \geq 0)$ of $u_{t}=u_{x x}+f\left(u, u_{x}\right)$ with $u(0, \cdot)=u_{0}$. The smoothing properties of the semiflow $\phi_{t}$ ensure that the orbit $u(t, \cdot)$ is precompact in $X$, and that the $\omega$-limit set $\omega\left(u_{0}\right)$ is a compact connected subset of the maximal compact attractor $A \subset X$ of the semiflow.

Our main results in this section are the following two theorems:

THEOREM 3.1. The $\omega$-limit set of any solution of (1.1) contains a rotating wave or a steady state.

COROLLARY 3.2. The only periodic orbits of (1.1) are rotating waves.

THEOREM 3.3. If all rotating waves and steady states of (1.1) are hyperbolic, then any orbit converges to exactly one of these rotating waves or steady states.

REMARK 3.1. If $U \in X$ is a homogeneous steady state of (1.1), i.e. $R_{\theta} U=U$ for all rotations $R_{\theta}$ (cf. (1.7)), then we call $U$ hyperbolic if the linearized flow $d \phi_{t}(U)$ has no eigenvalues on the unit circle $(0<t<\infty)$. If $U \in X$ is a standing wave (or a rotating wave with period $\tau>0$ ) then

$$
\left.\frac{d}{d \theta}\right|_{\theta=0} R_{\theta} U=U_{x} \not \equiv 0
$$

is always an eigenfunction of $d \phi_{\tau}(U), 0<\tau<\infty$ (resp. $\tau$ the period), with eigenvalue 1 . In that case, we call $U$ hyperbolic if this eigenvalue 1 is simple and (consequently, by Theorem 2.1) no other eigenvalues occur on the unit circle. For rotating waves $U$, this means that the periodic orbit $U(t, \cdot)=U(x-c t)$ is hyperbolic in the usual sense. For a standing wave $U$ it implies that the manifold $\left\{R_{\theta} U \mid 0 \leq \theta<2 \pi\right\}$ of shifted standing waves is normally hyperbolic in the sense of Hirsch, Pugh and Shub [25], see also [16].

REMARK 3.2. We tend to believe that Theorem 3.3 holds without the assumption of hyperbolicity. The autonomous reader is encouraged to reach a decisive answer. 
The proof of Theorem 3.1 will consist of a careful study of the zero number of the functions $u_{t}(t, \cdot)$ and $u_{x}(t, \cdot)$ for orbits $u(t, \cdot)$ in the maximal compact attractor A. Theorem 3.3 then follows from an analysis of the zero number on the (strong) stable (resp. unstable) manifold of rotating waves and steady states (cf. Lemma $3.8)$.

The next lemma is the key to the proof of Theorem 3.1. For each $u \in A$ let $E_{u} \subset X$ be the subspace spanned by $u_{t}$ and $u_{x}$. We are interested in those $u \in A$ for which $E_{u}$ has (real) dimension 1, i.e. one of $u_{t}, u_{x}$ is a multiple of the other. Then $u$ is a rotating wave $\left(u_{t}=c u_{x} \not \equiv 0\right)$, or a standing wave $\left(u_{t}=0, u_{x} \not \equiv 0\right)$, or a homogeneous nonstationary orbit $\left(u_{t} \neq 0, u_{x} \equiv 0\right)$. Let $u_{0} \in X$ be an arbitrary initial value, again.

LEMMA 3.4. If $u \in \omega\left(u_{0}\right)$ and $v \in E_{u} \backslash\{0\}$, then $v$ has only simple zeros.

PROOF. We argue by contradiction. Let $u \in \omega\left(u_{0}\right)$ and $v \in E_{u}$ be given such that $v$ has a multiple zero.

Since $\omega\left(u_{0}\right)$ is contained in the maximal attractor $\mathcal{A}$, there is an orbit $u(t, \cdot)$ $(t \in \mathbb{R})$ through $u \in \omega\left(u_{0}\right)$. By definition of $E_{u}$ there exist $\alpha, \beta \in \mathbb{R}$ such that $v(x)=\alpha u_{t}(0, x)+\beta u_{x}(0, x)$. We can define $v(t, \cdot)$ for all $t \in \mathbb{R}$ by $v(t, \cdot)=\alpha u_{t}(t, \cdot)+$ $\beta u_{x}(t, \cdot)$. Then $v(t, \cdot)$ is a solution of the linear parabolic equation

$$
v_{t}=v_{x x}+f_{p}\left(u, u_{x}\right) v_{x}+f_{u}\left(u, u_{x}\right) v \quad\left(x \in S^{1}, t \in \mathbf{R}\right) .
$$

Hence if $v$ has a multiple zero at $t=0$ then there exists $\delta>0$ such that $v$ has simple zeros at $t= \pm \delta$, and such that $z(v(\delta, \cdot))<z(v(-\delta, \cdot))$ holds (Theorem 5.1).

Now let $u^{0}(t, \cdot)=\phi_{t}\left(u_{0}\right) \in X$ denote the orbit through $u_{0}$, which is of course asymptotic to $\omega\left(u_{0}\right)$. Then there exists a large $t_{1}>0$ such that $u^{0}\left(t_{1}, \cdot\right)$ comes arbitrarily close to $u(\delta, \cdot)$ in the $C^{3}$ topology (we use the smoothing effect of the semiflow). Because $u_{t}^{0}=u_{x x}^{0}+f\left(u^{0}, u_{x}^{0}\right)$ holds along the orbit $u^{0}(t, \cdot)$, this implies that $v^{0}\left(t_{1}, \cdot\right)$ defined by $v^{0}\left(t_{1}, \cdot\right)=\alpha u_{t}^{0}\left(t_{1}, \cdot\right)+\beta u_{x}^{0}\left(t_{1}, \cdot\right)$ comes $C^{1}$ close to $v(\delta, \cdot)$. The upshot is that we can choose $t_{1}$ so large that $z\left(v^{0}\left(t_{1}, \cdot\right)\right)=z(v(\delta, \cdot))$. On the other hand the entire orbit $u(t, \cdot), t \in \mathbb{R}$ belongs to $\omega\left(u_{0}\right)$ so that we can choose a $t_{2}>t_{1}$ such that $u^{0}\left(t_{2}, \cdot\right)$ comes $C^{3}$ close to $u(-\delta, \cdot)$. As above, we conclude that $v^{0}\left(t_{2}, \cdot\right)$ comes $C^{1}$ close to $v(-\delta, \cdot)$ so that $z\left(v^{0}\left(t_{2}, \cdot\right)\right)=z(v(-\delta, \cdot))$. But now we have a contradiction, for $v^{0}(t, \cdot)$ solves the linear equation (3.1), replacing $u$ by $u^{0}$ there, so that $z(v(-\delta, \cdot))=z\left(v^{0}\left(t_{2}, \cdot\right)\right) \leq z\left(v^{0}\left(t_{1}, \cdot\right)\right)=z(v(\delta, \cdot))<z(v(-\delta, \cdot))$ holds.

COROLlARY 3.5. If $u \in \omega\left(u_{0}\right), v, w \in E_{u}$ and $v$ and $w$ have a common zero then one is a multiple of the other.

PROOF. Indeed, if $v\left(x_{0}\right)=w\left(x_{0}\right)=0$ for some $x_{0} \in S^{1}$, then $v_{x}\left(x_{0}\right) \cdot w-w_{x}\left(x_{0}\right) \cdot v$ lies in $E_{u}$ and has a multiple zero at $x_{0}$. Hence $v_{x}\left(x_{0}\right) \cdot w-w_{x}\left(x_{0}\right) \cdot v \equiv 0$ by Lemma 3.4. If $v_{x}\left(x_{0}\right)=0$ then $v \equiv 0$, again by Lemma 3.4. Otherwise $w=$ $\left(w_{x}\left(x_{0}\right) / v_{x}\left(x_{0}\right)\right) \cdot v$.

We can now prove Theorem 3.1. In fact we show a slightly stronger statement.

THEOREM 3.6. If $C \subset \omega\left(u_{0}\right)$ is a nonempty closed, flow invariant subset, then $C$ contains a rotating wave or a steady state.

PrOOF. Consider the function from $C \times S^{1}$ to $\mathbf{R}$ given by point evaluation: $e: C \times S^{1} \rightarrow \mathbb{R},(u, x) \rightarrow u(x)$. The function $e$ is continuous and $C \times S^{1}$ is compact, so $e$ achieves its maximum at some $(U, x) \in C \times S^{1}$. 
It follows that $U_{x}(x)=0$, and by flow invariance of $C$ also that $U_{t}(x)=0$.

But then $U_{t}$ and $U_{x}$ in $E_{u}$ have a common zero, so that they are linearly dependent. Hence $U$ is a rotating wave or a steady state by the remarks preceding Lemma 3.4. This proves Theorems 3.6 and 3.1.

In the remaining part of this section we shall prove Theorem 3.3. We therefore assume that all steady states and rotating waves are hyperbolic (cf. Remark 3.1).

Let $U \in A$ be a steady state, i.e. let $U(x)$ be any solution of

$$
U_{x x}+f\left(U, U_{x}\right)=0, \quad U(x+2 \pi) \equiv U(x) .
$$

Recall the definition [20] of the local strong stable and strong unstable manifold of $U$. These smooth manifolds consist of those solutions $u(t, \cdot)$ which approach $U$ exponentially fast as $t \rightarrow+\infty$ (resp. $t \rightarrow-\infty$ ), without leaving a fixed small neighborhood of $U$. These manifolds extend globally, as usual. Here we use backward uniqueness which holds in our case by real analyticity; see [20, Chapter 6]. In case $U$ is homogeneous they coincide with the usual (local) stable and unstable manifold of $U$.

Let $T(t)=d \phi_{t}(U)$ be the linearization of the semiflow at $U$. By Theorem 2.1, the spectrum of $T(t)$ is given by $\lambda_{j}=\exp \left(t \mu_{j}\right), j=0,1,2, \ldots$ with $\operatorname{Re} \mu_{j} \geq \operatorname{Re} \mu_{j+1}$ and $\operatorname{Re} \mu_{2 j}>\operatorname{Re} \mu_{2 j+1}$. The instability index $i(U)$ of $U$ was defined as the number of $j$ with $\operatorname{Re} \mu_{j}>0$ (or $\left|\lambda_{j}\right|>1$ ).

Our main tool in the proof of Theorem 3.3 is the next result.

LEMMA 3.7. Let $U$ be a hyperbolic steady state. Then $z\left(u_{0}-U\right) \leq i(U)$ for any $u_{0}$ in the strong unstable manifold of $U$. If $u_{0} \not \equiv U$ lies in the strong stable manifold of $U$, then $z\left(u_{0}-U\right) \geq i(U)+1$.

Proof. The idea of the proof is the following. Consider $v(t, \cdot):=u(t, \cdot)-U$ along the semiflow $u(t, \cdot)$ through $u_{0}$. Note that $v$ satisfies an equation

$$
v_{t}=v_{x x}+g\left(x, v, v_{x}\right)
$$

with $g(x, v, p):=f\left(U(x)+v, U_{x}(x)+p\right)-f\left(U(x), U_{x}(x)\right)$. Because $g(x, 0,0)=0$, the zero number $z(v(t, \cdot))$ is nonincreasing with $t$. Basically, we expect $v(t, \cdot) /\|v(t, \cdot)\|_{X}$ to approach an "eigenspace" $E_{2 j}$ for the linearized semiflow as $t \rightarrow \pm \infty$. Then our result follows because $z=2 j$ on $E_{2 j}$, by Theorem 2.2 . We shall consider the technically more delicate case of the strong stable manifold, only, and leave the other case to the reader.

Define

$$
\omega=\max \left(\operatorname{Re} \mu_{j}: \operatorname{Re} \mu_{j}<0\right) .
$$

Then $\left\{\exp t \mu_{j}: \operatorname{Re} \mu_{j}=\omega\right\}$ is a spectral set for $T(t)$ and we denote the corresponding spectral projection in $X$ by $P$. It follows from Theorem 2.2 that any nonzero $v \in \operatorname{Range}(P) \backslash\{0\}$ satisfies $z(v) \geq i(U)+1$.

More precisely, if $U$ is a homogeneous steady state, then $i(U)$ is odd and Range $(P)=E_{i(U)+1}$ in the notation of $\S 2$. Alternatively, if $U$ is a standing wave, then $\lambda=1$ is an eigenvalue so that $i(U)$ may be odd or even: if $i(U)=2 N-1$, then the eigenvalues $\lambda_{2 N-1}=1$ and $\lambda_{2 N}$ form a spectral set in the sense of Theorem 2.1 , and Range $P$ is the eigenspace belonging to $\lambda_{2 N}$, hence Range $P \subseteq E_{2 N}$. If $i(U)=2 N$, then one either has Range $(P)=E_{2 N+2}\left(\right.$ when $\left.\left|\lambda_{2 N+1}\right|=\left|\lambda_{2 N+2}\right|\right)$ or Range $(P)$ is the eigenspace belonging to $\lambda_{2 N+1}$ (when $\left|\lambda_{2 N+1}\right|>\left|\lambda_{2 N+2}\right|$ ), 
Range $P \subseteq E_{2 N+2}$. In each case one sees that indeed $z(v) \geq i(U)+1$ by Theorem 2.2 .

From here on, the proof is analogous to the arguments given in [7]. For the convenience of the reader, we sketch the line of reasoning. On the strong stable manifold, the difference $v=u-U$ tends to zero for $t \rightarrow \infty$. For $v /\|v\|_{X}$ we have the following alternative:

$$
\lim _{t \rightarrow \infty} \frac{\|P v\|}{\|v\|}=0
$$

or

$$
\lim _{t \rightarrow \infty} \frac{\|(\mathrm{id}-P) v\|}{\|v\|}=0 .
$$

Case (a) holds for $v$ in a submanifold of codimension 1 (within the local strong stable manifold $W^{\mathrm{ss}}$ ) which we call the fast stable manifold $W^{\mathrm{fs}}$. Case (b) holds for the remaining $v$ in $W^{\text {ss }}$. For a reference see $[7,16]$.

Assume that case (b) holds. Then

$$
\lim _{t_{n} \rightarrow \infty} \frac{v\left(t_{n}, \cdot\right)}{\left\|v\left(t_{n}, \cdot\right)\right\|}=w \in \text { Range } P \backslash\{0\}
$$

exists for a subsequence $t_{n} \rightarrow \infty$. Applying Theorem 2.2 to the above discussion of Range $P$ we see that $w$ (and likewise $v\left(t_{n}, \cdot\right)$ for $t_{n}$ large) has at least $i(U)+1$ zeros (all of them being simple).

If case (a) holds, then $v(t, \cdot)$ has only simple zeros for most $t>0$ (cf. Corollary 5.3 , or $[6])$. But then $z(v(t, \cdot))=z(\tilde{v})$ for any $\tilde{v}$ near $v$. Picking $\tilde{v}$ in $W^{\text {ss }} \backslash W^{\mathrm{fs}}$, case (a) applies to $\tilde{v}$ and

$$
z(v(0, \cdot)) \geq z(v(t, \cdot))=z(\tilde{v}) \geq i(U)+1,
$$

completing the proof.

PROOF OF THEOREM 3.3. The proof is indirect and will be given in three steps. We begin with an outline. Let $u_{0} \in X$ be an initial value with $\omega$-limit set $\omega\left(u_{0}\right)$. By Theorem 3.1, $\omega\left(u_{0}\right)$ contains a steady state or a rotating wave $U$, which is hyperbolic by assumption. In step 1 below we show that we may assume $U$ to be a steady state, without loss of generality. Assuming $\{U\} \subsetneq \omega\left(u_{0}\right), \omega\left(u_{0}\right)$ has to contain orbits $V^{+} \not \equiv R_{\theta}(U)$ resp. $V^{-} \not \equiv R_{\theta^{\prime}}(U)$ in the strong stable manifold $W_{\theta}^{\text {ss }}$ of $R_{\theta}(U)$ (resp. the strong unstable manifold $W_{\theta^{\prime}}^{\text {su }}$ of $R_{\theta^{\prime}}(U)$ ) for suitable, possibly different $\theta, \theta^{\prime}$ (step 2). Since the linearizations about $R_{\theta}(U)$ and $R_{\theta^{\prime}}(U)$ have the same spectrum, Lemma 3.7 tells us that

$$
z\left(V^{-}-R_{\theta}(U)\right)<z\left(V^{+}-R_{\theta^{\prime}}(U)\right) .
$$

In Step 3 we prove that for any steady state $\hat{U}$ and for any $V \in \omega\left(u_{0}\right) \backslash\{\hat{U}\}$ the difference $V-\hat{U}$ has only simple zeros, and $z(V-\hat{U})$ is independent of the choice of $V \in \omega\left(u_{0}\right) \backslash\{\hat{U}\}$. Picking $V:=V^{+}$and $\hat{U}:=R_{\sigma}(U)$ for $\sigma$ from $\theta$ to $\theta^{\prime},(3.3)$ implies

$$
z\left(V^{-}-R_{\theta}(U)\right)<z\left(V^{+}-R_{\theta}(U)\right)
$$

On the other hand $V^{+}$and $V^{-}$are both in $\omega\left(u_{0}\right) \backslash\left\{R_{\theta}(U)\right\}$. Hence step 3 also implies

$$
z\left(V^{-}-R_{\theta}(U)\right)=z\left(V^{+}-R_{\theta}(U)\right)
$$


a contradiction to (3.4). Therefore $\{U\}=\omega\left(u_{0}\right)$. It remains to prove steps $1-3$.

Step 1 . Without loss of generality, $\omega\left(u_{0}\right)$ contains a hyperbolic steady state $U$.

Otherwise, $\omega\left(u_{0}\right)$ contains a rotating wave $U$ with speed $c \neq 0$ (Theorem 3.1). Consider the semiflow $\psi_{t}:=R_{c t} \phi_{t}$ generated by the parabolic equation

$$
u_{t}=u_{x x}+c u_{x}+f\left(u, u_{x}\right) \text {. }
$$

On the circle $R_{\theta}(U), 0 \leq \theta<2 \pi$, each $R_{\theta}(U)$ is a standing wave for the semillow $\psi_{t}$. Moreover there exists a $\theta$ such that $R_{\theta}(U) \in \omega\left(\psi_{t}\left(u_{0}\right)\right)$ by definition of the $\omega$-limit set; we may assume that $\theta=0$ without loss of generality. So $U$ is a standing wave for $\psi_{t}$, but a rotating wave of (not necessarily minimal) period $\tau=2 \pi / c$ for $\phi_{t}$. Being hyperbolic for $\phi_{t}, U$ remains hyperbolic for $\psi_{t}$; in the notation of $\S 2$ :

$$
d \psi_{\tau}(U)=d\left(R_{c \tau} \phi_{\tau}(U)\right)=R_{c \tau} d \phi_{\tau}(U)=d \phi_{\tau}(U)
$$

since $c \tau=2 \pi$ and $R_{2 \pi}=$ id. Now suppose we have shown that $\omega\left(\psi_{t}\left(u_{0}\right)\right)=\{U\}$. For $\phi_{t}$ this implies

$$
\omega\left(u_{0}\right)=\omega\left(\phi_{t}\left(u_{0}\right)\right) \subseteq \bigcup_{\theta \in S^{1}} R_{\theta} \omega\left(\psi_{t}\left(u_{0}\right)\right)=\left\{R_{\theta} U: \theta \in S^{1}\right\},
$$

i.e. $\omega\left(u_{0}\right)$ consists of the single rotating wave $U$. This proves step 1 .

Step 2. Suppose $\{U\} \subsetneq \omega:=\omega\left(u_{0}\right)$ for some steady state $U$. Then there exist

$$
V^{+} \in\left(W_{\theta}^{\text {ss }} \cap \omega\right) \backslash\left\{R_{\theta}(U)\right\}, \quad V^{-} \in\left(W_{\theta^{\prime}}^{\text {su }} \cap \omega\right) \backslash\left\{R_{\theta^{\prime}}(U)\right\}
$$

for suitably chosen $\theta$ and $\theta^{\prime}$.

If $\omega$ contains anything besides $U$ then, by normal hyperbolicity of the point or circle $R U:=\left\{R_{\theta} U: \theta \in S^{1}\right\}$ (cf. [25], Remark 3.1, and [20, p. 108]), there exists a neighborhood $\mathcal{N}$ of $R U$ such that $\omega \backslash \overline{\mathcal{N}}$ is nonempty. Hence there is a sequence $t_{n} \uparrow \infty$ such that $\phi_{t_{n}}\left(u_{0}\right) \rightarrow U$ in $X$, and such that the orbit $\phi_{t}\left(u_{0}\right)$ leaves $\overline{\mathcal{N}}$ in each time interval $t_{n} \leq t \leq t_{n+1}$. Define

$$
\begin{aligned}
& s_{n}:=\sup \left(s \in\left(t_{n-1}, t_{n}\right): \phi_{s}\left(u_{0}\right) \in X \backslash \overline{\mathcal{N}}\right), \\
& u_{n}:=\phi_{s_{n}}\left(u_{0}\right) .
\end{aligned}
$$

Then all $u_{n}$ lie on $\partial \mathcal{N}$, and because the $s_{n}$ tend to infinity as $n \rightarrow \infty$ we may extract a subsequence of the $u_{n}$ which converges to a $V^{+} \in \omega\left(u_{0}\right)$.

By construction we have $\phi_{t_{n}-s_{n}}\left(u_{n}\right) \rightarrow U$ as $n \rightarrow \infty$ so that $t_{n}-s_{n} \rightarrow \infty$ as $n \rightarrow \infty$ (otherwise we could take a subsequence such that $t_{n}-s_{n} \rightarrow t^{*}$ and obtain $\phi_{t^{*}}\left(V^{+}\right)=U$, which is clearly impossible). Therefore the orbit $\phi_{t}\left(V^{+}\right), t \geq 0$, is contained in $\overline{\mathcal{N}}$. By normal hyperbolicity [25] of the set $R U$ of steady states, this orbit lies in the local strong stable manifold $W_{\theta}^{\text {ss }}$ of some single $R_{\theta} U$; cf. [20, p. 108]. Of course, $V^{+} \not \equiv R_{\theta} U$ because $V^{+} \in \partial \mathcal{N}$.

A similar argument shows the existence of $V^{-}$.

Step 3 . For any steady state $\hat{U}$ and for any $V \in \omega\left(u_{0}\right) \backslash\{\hat{U}\}$ the difference $V-\hat{U}$ has only simple zeros and $z(V-\hat{U})$ is independent of the choice of $V$.

Indeed, $z(t):=z(u(t, \cdot)-\hat{U})$ is nonincreasing along $u(t, \cdot)=\phi_{t}\left(u_{0}\right)$ so that $z(t)$ is constant, say, for $t \geq t_{1}$. If $V-\hat{U}$ had a multiple zero then $z\left(\phi_{t}(V)-\hat{U}\right)$ would strictly decrease at $t=0$ by Theorem 5.1 . However we can find a large $t_{2} \geq t_{1}$ such that the orbit segment $\left\{u\left(t_{2}+t, \cdot\right)\right\}_{|t| \leq 1}$ comes arbitrarily close to $\left\{\phi_{t}(V)\right\}_{|t| \leq 1}$ so 
that $z\left(u\left(t_{2}+t, \cdot\right)-\hat{U}\right)$ would have to drop at some $t$ close to zero. This contradicts the fact that $z(t)$ is constant for $t \geq t_{1}$.

It is clear from the above argument that $z(V-\hat{U})$ equals the eventual value of $z(u(t, \cdot)-\hat{U})$, i.e. does not depend on $V$. This completes the proof of Theorem 3.3 .

4. Connections. Let $U$ be a hyperbolic steady state or a hyperbolic rotating wave of our semiflow $\left\{\phi_{t}\right\}_{t \geq 0}$ (cf. Remark 3.1). In this section we shall consider the problem of determining the asymptotic behavior of orbits on the strong unstable manifold $W^{\text {su }}$ of $U$.

Recall that $i(U)$ was defined to be the number of eigenvalues $\lambda$ of $d \phi_{\tau}(U)$ with $|\lambda|>1$, counted with algebraic multiplicity. We shall assume that $i(U)>0$ (cf. Corollary 2.4). Define $w_{ \pm} \in X$ by

$$
\begin{aligned}
& w_{+}=\inf (w>U: w \text { is a steady state }) \\
& w_{-}=\sup (w<U: w \text { is a steady state }) .
\end{aligned}
$$

Then we have

THEOREM 4.1. Let $U$ be a steady state with $i(U)>0$ or a rotating wave. Then $w_{+}$and $w_{-}$are well defined. They are homogeneous steady states satisfying $w_{-}<U<w_{+}, i\left(w_{ \pm}\right)=0$, and $U$ connects to both $w_{+}$and $w_{-}$.

This result seems to be well known and holds for much more general types of equations, cf. the type of reasoning in $[\mathbf{2 3}, \mathbf{2 4}]$ and, especially, [37, Theorem 8]. We sketch the proof. As in the proof of Theorem 3.3, step 1, we may assume $U$ is a steady state, without loss of generality. Since $i(U)>0$, the largest eigenvalue $e^{\mu_{0} t}$ of $d \phi_{t}(U)$ satisfies $e^{\mu_{0} t}>1$. Let $u(t, \cdot)$ be an orbit on the corresponding one-dimensional fast unstable manifold (cf. [7]). Then

$$
\psi=\lim _{t \rightarrow-\infty} \frac{u_{t}(t, \cdot)}{\left\|u_{t}(t, \cdot)\right\|}
$$

is the first eigenfunction of $d \phi_{t}(U)$, so that either $\psi>0$ or $\psi<0$. Suppose for convenience that $\psi>0$. It follows that $u_{t}(t, \cdot)>0$ for all $t \in \mathbb{R}$ because $z\left(u_{t}(t, \cdot)\right)$ is nonincreasing. As we have assumed the existence of a maximal compact attractor by $(1.2 \mathrm{a}, \mathrm{b}), u_{+}:=\sup _{t} u(t, \cdot)$ exists. It is easily verified that $u_{+}$is a steady state, and that any steady state $v>u$ also satisfies $v \geq u_{+}$. Hence $u_{+}=w_{+}$and $u$ connects to $w_{+}$via the orbit $u(t, \cdot)$. Finally note that $w_{+}=u_{+}$is stable from below, hence $d \phi_{t}\left(w_{+}\right)$cannot have eigenvalues outside of the unit circle. Taking $\psi<0$, we similarly obtain a connection to $w_{-}$.

Our main result in this section is the following.

THEOREM 4.2. Let $U$ be a hyperbolic steady state, or a hyperbolic rotating wave, with unstable dimension $i(U)$. Then for any $k \in \mathbb{N}, 0<2 k \leq i(U)$, there exists a rotating wave or steady state $w_{k}$ such that $w_{-}<w_{k}<w_{+}, z\left(w_{k}-U\right)=2 k$, $U$ connects to $w_{k}$.

Before we dive into the proof, we give a few comments. Because $U$ is assumed to be hyperbolic, $U$ connects to $w_{k}$ iff there exists an orbit $u(t, \cdot)$ in the strong unstable manifold $W^{\mathrm{su}}$ of $U$ such that $w_{k} \in \omega(u(t, \cdot))$ (cf. the proof of Theorem $3.3)$. 
If all steady states and rotating waves are assumed to be hyperbolic, then $\omega(u(t, \cdot))$ consists just of $w_{k}$ by Theorem 3.3. However, this does not imply uniqueness of the $w_{k}$ : given $k$ as above, there might exist several $w_{k}$ complying with the conclusions of Theorem 4.2.

Invoking Theorem 3.1 , it will be sufficient to obtain a trajectory $u(t, \cdot)$ in the (strong) unstable manifold $W^{\text {su }}$ of $U$ such that

$$
z(t):=z(u(t, \cdot)-U(t, \cdot)) \equiv 2 k,
$$

for all $t \in \mathbf{R}$. Along our way, we will in fact prove that the function $z(t)$ may be prescribed arbitrarily (with $z(t)$ even, nonincreasing and $z(t) \leq i(U)$, of course), and still there exists a trajectory $u(t, \cdot)$ in $W^{\text {su }}$ realizing this given graph of $z$. We single out this result in Theorem 4.3 below.

As was mentioned in the introduction, Theorem 4.2 shows some similarity with results in [8] and [33]. In fact the main strategy of the proof follows [8], but the underlying topology is different due to the occurrence of periodic orbits viz. complex eigenvalues.

The main idea of the proof is the following. Replacing the flow $\phi_{t}$ by $\psi_{t}:=$ $R_{-c t} \phi_{t}$, if necessary, we may assume that $U$ is a hyperbolic steady state (see the proof of Theorem 3.3, step 1). For any orbit $u \not \equiv U$ in the maximal compact attractor $A$ we then define the dropping times

$$
t_{n}:=\inf \{t \in \mathbf{R}: z(t) \leq 2 n\}
$$

(thus $\left.-\infty \leq t_{n} \leq+\infty\right)$, where $z(t)=z(u(t, \cdot)-U)$. Recall that $v:=u-U$ satisfies the equation

$$
v_{t}=v_{x x}+g\left(x, v, v_{x}\right)
$$

with $g(x, v, p):=f\left(U(x)+v, U_{x}(x)+p\right)-f\left(U(x), U_{x}(x)\right)$. Because $g(x, 0,0)=0$, $z(t)$ is nonincreasing in $t$. Moreover $z(t) \leq i(U)$ for orbits $u$ in the strong unstable manifold $W^{\text {su }}$ of $U$, hence $-\infty=t_{N} \leq t_{N-1} \leq \cdots \leq t_{1} \leq t_{0} \leq+\infty$ where $N$ is the largest integer such that $2 N \leq i(U)$.

These dropping times $t_{n}$ can be regarded as functions of the orbit $u(t, \cdot)$ on the strong unstable manifold.

If we then choose $t_{N}=t_{N-1}=\cdots=t_{k}=-\infty$ and $t_{k-1}=t_{k-2}=\cdots=t_{0}=$ $+\infty$ (for some integer $k$ with $0<2 k \leq i(U)$ ), we get an orbit $u$ with $z(u(t, \cdot)-U)=$ $2 k$ for all $t \in \mathbf{R}$, which shows that Theorem 4.2 is true.

We proceed to fill in the details. For the time being we shall assume that $i(U)=2 N+1$ is odd, deferring the case that $i(U)=2 N$ is even to the end of this section. Let $\Sigma^{2 N}$ be a small $2 N$-sphere of radius $\varepsilon>0$ in the strong unstable manifold $W^{\text {su }}$ of $U$.

DEFINITION OF THE $y$-MAP. We shall now define a map $y: \Sigma^{2 N} \rightarrow \mathbf{R} \times \mathbb{C}^{N}$ which encodes the dependence of the dropping times on the orbit $u$ in $W^{\text {su }}$. Let $u_{0} \not \equiv U$ with $z\left(u_{0}\right) \leq 2 N$ be given, and let $u(t, \cdot)$ be the corresponding orbit with $u(0, \cdot)=u_{0}$ and dropping times denoted by $t_{n}\left(u_{0}\right)$. It will be convenient to consider

$$
\tau_{n}\left(u_{0}\right)=\frac{1}{2} \tanh t_{n}\left(u_{0}\right) \quad(0 \leq n \leq N)
$$

(with $\tanh ( \pm \infty)= \pm 1$ ), instead of the $t_{n}\left(u_{0}\right)$.

Furthermore for each pair $t_{n}<t_{n-1}$ we shall need a phase angle $\theta_{n} \in \mathbf{R} / 2 \pi \mathbb{Z}$. Technically, our seemingly arbitrary, but crucial choice for $\theta_{n}$ will be justified by 
Theorem 4.3, in particular cf. (4.1). The role of $\theta_{n}$ is analogous to the sign $\sigma_{n}$ of $y_{n}$ chosen in $[8,(2.3)]$. But our choice is not the only possible one. We define this angle $\theta_{n}$ as follows: if $\tau_{n}\left(u_{0}\right)<\tau_{n-1}\left(u_{0}\right)$ put

$$
s_{n}\left(u_{0}\right)=\tanh ^{-1}\left[\left(\tau_{n}\left(u_{0}\right)+\tau_{n-1}\left(u_{0}\right)\right)\right] \quad(1 \leq n \leq N)
$$

and let $\theta_{n}\left(u_{0}\right)$ be the sum (in the group $\mathbb{R} / 2 \pi \mathbb{Z}$ ) of the zeros $x_{j}$ of the function $x \rightarrow u\left(s_{n}\left(u_{0}\right), x\right)-U(x)$ on $S^{1}$. If $\tau_{n}<\tau_{n-1}$, these zeros are simple (Theorem 5.1), hence their sum will always be finite. Put $\theta_{n}\left(u_{0}\right)=1$, if $\tau_{n}\left(u_{0}\right)=\tau_{n-1}\left(u_{0}\right)$.

Finally we need a sign $\sigma\left(u_{0}\right) \in\{ \pm 1\}=S^{0}$ to define the $y$-map. This sign is given by

$$
\sigma\left(u_{0}\right)=\left\{\begin{array}{l}
0 \text { if } t_{0}=+\infty \\
\operatorname{sign}(u(t, \cdot)-U(\cdot)) \quad \text { for some } t>t_{0} \text { if } t_{0}<+\infty .
\end{array}\right.
$$

Note that the function $u(t, \cdot)-U(\cdot)$ has no zeros for $t>t_{0}$ so that the sign $\sigma\left(u_{0}\right)$ is well defined.

The $y$-map can now be defined as follows:

$$
\begin{aligned}
& y_{0}\left(u_{0}\right):=\sigma\left(u_{0}\right) \cdot\left(\frac{1}{2}-\tau_{0}\left(u_{0}\right)\right)^{1 / 2}, \\
& y_{n}\left(u_{0}\right)=e^{i \theta_{n}\left(u_{0}\right)}\left(\tau_{n-1}\left(u_{0}\right)-\tau_{n}\left(u_{0}\right)\right)^{1 / 2} \quad \text { for } 1 \leq n \leq N,
\end{aligned}
$$

and

$$
y\left(u_{0}\right)=\left(y_{0}\left(u_{0}\right), y_{1}\left(u_{0}\right), \ldots, y_{N}\left(u_{0}\right)\right) \in \mathbb{R} \times \mathbb{C}^{N} .
$$

Using the fact that $t_{N}\left(u_{0}\right) \equiv-\infty$, i.e. that $\tau_{N}\left(u_{0}\right) \equiv-\frac{1}{2}$ we see that for any $u_{0} \not \equiv 0$ with $z\left(u_{0}\right) \leq 2 N$

$$
\sum_{n=0}^{N}\left|y_{n}\left(u_{0}\right)\right|^{2}=\frac{1}{2}-\tau_{0}+\sum_{1}^{N}\left(\tau_{n-1}-\tau_{n}\right)=1
$$

holds, so that $y$ is a map from $\Sigma^{2 N}$ into the unit sphere $S^{2 N}$ in $\mathbb{R} \times \mathbb{C}^{N}$.

If $y: \Sigma^{2 N} \rightarrow S^{2 N}$ is surjective, then for any sequence of dropping times there exists an orbit $u(\cdot)$ with these dropping times by the definition of the $y$-map. We want to prove that $y: \Sigma^{2 N} \rightarrow S^{2 N}$ is surjective.

THEOREM 4.3. The map $y: \Sigma^{2 N} \rightarrow S^{2 N}$ defined above is essential, i.e. not homotopic to a constant map. In particular, $y$ is surjective. Thus for any nonincreasing sequence $\infty \geq t_{0}^{\prime} \geq t_{1}^{\prime} \geq \cdots \geq t_{N}^{\prime}=-\infty$ there exists an orbit $u(t, \cdot)$ in the strong unstable manifold of the steady state $U$ with $u_{0}=u(0, \cdot) \in \Sigma^{2 N}$ such that $t_{k}\left(u_{0}\right)=t_{k}^{\prime}$ for all $k$ with $0 \leq k \leq N$.

REMARK 4.1. This theorem, as stated here, holds for completely arbitrary (but analytic) nonlinearities $f=f\left(x, u, u_{x}\right)$ for which (1.1) defines a global semiflow. It is the rotating waves aspect, which made us restrict our attention to the $S^{1}$ equivariant setting $f=f\left(u, u_{x}\right)$.

PROOF OF THEOREMS 4.2 AND 4.3 (FOR $i(U)=2 N+1$ ). The proof has three ingredients. First we discuss continuity of the $y$-map. Then we give a homotopy from the nonlinear equation satisfied by $u(t, \cdot)-U$ to a standard linear heat equation. Invoking a theorem by Hopf and Rueff [26] will prove that the accordingly deformed $y$-map, and hence the original $y$-map, is essential. The remaining statements of the theorem are then immediate. 
We give a brief account of continuity of the $y$-map. For more details see [8, §2]. The transformed dropping times $\tau_{n}$ depend continuously on $u_{0}$ because $v=u-U$ has only simple zeros before and after each dropping of $z, \mathrm{cf}$. [6], and also [36]. If $\tau_{n}<\tau_{n-1}$, then the phase angle $\theta_{n} \in S^{1}$ also depends continuously on $u_{0}$, again because the zeros $x_{j}$ which define $\theta_{n}$ are all simple. If on the other hand $\tau_{n-1}\left(u_{0}\right)=$ $\tau_{n}\left(u_{0}\right)$, then $\theta_{n}$ may be discontinuous at $u_{0}$. But $y_{n}\left(u_{0}\right)$ is still continuous at $u_{0}$, due to the factor $\left(\tau_{n-1}-\tau_{n}\right)^{1 / 2}$. The argument for the sign $\sigma\left(u_{0}\right)$ and $y_{0}\left(u_{0}\right)$ is analogous.

Recall that $y$ was defined via

$$
v_{t}=v_{x x}+g\left(x, v, v_{x}\right)
$$

which is satisfied by $v=u-U$. Thus $y$ also depends on $g$. This dependence is continuous, if we consider $g \in C^{k}\left(S^{1} \times \mathbb{R}^{2}, \mathbb{R}\right)$ and endow $C^{k}$ with the compact open topology (i.e. with the weak Whitney topology [22]). Of course we need $g(x, 0,0)=0$ and a growth condition like $(1.2 \mathrm{~b})$ in order to define $y$. For more details, see $[8, \S 2]$ again.

We construct the promised homotopy to linear $g$ next. We split this homotopy into two parts: first we follow

$$
g^{\theta}(x, u, p):=(1-2 \theta) g(x, u, p)+2 \theta(a(x) p+b(x) u) \quad\left(0 \leq \theta \leq \frac{1}{2}\right)
$$

with $a(x):=g_{p}(x, 0,0), b(x):=g_{u}(x, 0,0)$, and after that

$$
g^{\theta}(x, u, p):=2(1-\theta)(a(x) p+b(x) u) \quad\left(\frac{1}{2} \leq \theta \leq 1\right)
$$

Note that $g^{1 / 2}$ is the linearization of $g^{0}=g$ at $u=p=0$, and $g^{1} \equiv 0$ provides the standard heat equation.

What happens to our $y$-map during this homotopy? Consider $0 \leq \theta \leq \frac{1}{2}$, first. During this homotopy, the strong unstable manifold $W^{\text {su }}$ of $U$ (alias 0 in the equation for $v=u-U$ ) is flattened until it coincides with its tangent space

$$
T W^{\mathrm{su}}(0)=E_{0} \oplus E_{2} \oplus \cdots \oplus E_{2 N}
$$

at zero. Here we use the notation of $\S 2$ freely. Choosing the radius $\varepsilon$ of the sphere $\Sigma^{2 N}$ small enough, we simultaneously obtain a homotopy of homeomorphisms of $\varepsilon$-spheres $\Sigma_{\theta}^{2 N}$ to an $\varepsilon$-sphere in the tangent space $T W^{\text {su }}(0)$, given by the eigenprojection from $W^{\text {su }}$ to $T W^{\text {su }}(0)$; for complete details see $[8, \S 2]$. This provides us with a homotopy $y^{\theta}$ of $y$-maps belonging to $g^{\theta}$.

It remains to show that $y^{1 / 2}$ is essential. This is done via $\frac{1}{2} \leq \theta \leq 1$. By Theorem 2.1 each $E_{2 j}$ varies continuously during this second homotopy-and so does the $y$-map. This continues the homotopy $y^{\theta}$ of $y$-maps to $\frac{1}{2} \leq \theta \leq 1$.

It remains to show that $y^{1}$, associated to the standard heat equation, is essential. For convenience we write $y, \Sigma^{2 N}$ for $y^{1}, \Sigma_{1}^{2 N}$. Recalling that

$$
E_{0}=\{\xi \mid \xi \in \mathbb{R}\}, \quad E_{2 j}=\left\{\xi_{j} \cos j x+\eta_{j} \sin j x \mid \xi_{j}, \eta_{j} \in \mathbf{R}\right\}
$$

for the linear heat equation, we may identify $E_{0} \oplus E_{2} \oplus \cdots \oplus E_{2 N}$ with $\mathbf{R} \times \mathbb{C}^{N}$ by

$$
\begin{aligned}
\xi+\sum_{j=1}^{N} \xi_{j} \cos j x-\eta_{j} \sin j x & \cong\left(\xi, \xi_{1}+i \eta_{1}, \ldots, \xi_{N}+i \eta_{N}\right) \\
& =\left(\xi, \varsigma_{1}, \ldots, \varsigma_{N}\right)=(\xi, \varsigma), \quad \varsigma_{j} \in \mathbb{C}, \varsigma \in \mathbb{C}^{N} .
\end{aligned}
$$


The action of $R_{\theta}$ in $\mathbb{R} \times \mathbb{C}^{N}$ is then given by

$$
R_{\theta}\left(\xi, \varsigma_{1}, \varsigma_{2}, \ldots, \varsigma_{N}\right)=\left(\varsigma, \alpha \varsigma_{1}, \alpha^{2} \varsigma_{2}, \ldots, \alpha^{N} \varsigma_{N}\right),
$$

where $\alpha=e^{i \theta}, 0 \leq \theta<2 \pi$.

We now claim that $y=y(\xi, \varsigma)=\left(y_{0}, y_{1}, \ldots, y_{N}\right)(\xi, \varsigma)$ has the following equivariance property:

$$
y\left(\sigma \xi, \alpha \varsigma_{1}, \alpha^{2} \zeta_{2}, \ldots, \alpha^{N} \varsigma_{N}\right)=\left(\sigma y_{0}, \alpha^{2} y_{1}, \alpha^{4} y_{2}, \ldots, \alpha^{2 N} y_{N}\right)(\xi, \varsigma)
$$

for any $\sigma \in(-1,1)$ and $\alpha \in \mathbb{C}$ with $|\alpha|=1$. This equivariance property is the point of the second homotopy $\frac{1}{2} \leq \theta \leq 1$. It does not hold for $x$-dependent $g$. To prove (4.1) we use that $y$ belongs to the equation $v_{t}=v_{x x}$. If we therefore rotate the initial value $v_{0}$ by $R_{\theta}$ the zeros of the corresponding orbit $v$ also get rotated by $\theta$. Since the phase of $y_{n}(1 \leq n \leq N)$ is given by the sum of $2 n$ of such zeros, the rotation has the effect of rotating $y_{n} \in \mathbb{C}$ by $2 n \theta$.

Concerning the sign of $\xi$ and $y_{0}$ above, we note that the orbit through $\xi+$ $\Sigma\left[\xi_{j} \cos j x-\eta_{j} \sin j x\right]$ converges to the constant $\xi \neq 0$ as $t \rightarrow \infty$. Hence we have $\operatorname{sign}\left(y_{0}\right)=\operatorname{sign}(\xi)$.

Thus $y$ is equivariant in the indicated manner. The preceding arguments also show that $y$ maps the upper hemisphere $\{\xi \geq 0\}$ in $\Sigma^{2 N}$ into the upper hemisphere $\left\{y_{0} \geq 0\right\}$ of $S^{2 N} \subset \mathbb{R} \times \mathbb{C}^{k}$, and also maps the corresponding lower hemisphere $\{\xi \leq 0\}$ into $\left\{y_{0} \leq 0\right\}$. Recall that, due to a theorem by H. Hopf $y: \Sigma^{2 N} \rightarrow S^{2 N}$ is essential iff its topological degree is nonzero. See e.g. $[\mathbf{1 4}, \mathbf{1 5}, \mathbf{1 8}, \mathbf{2 2}, \mathbf{3 4}]$ for a background on the topological facts used. For the equators $\Sigma^{2 N-1}=\{\xi=0\} \cap \Sigma^{2 N}$ and $S^{2 N-1}=\left\{y_{0}=0\right\} \cap S^{2 N}$ we obtain a map

$$
Y: \Sigma^{2 N-1} \rightarrow S^{2 N-1}, \quad \varsigma \rightarrow\left(y_{1}(0, \varsigma), \ldots, y_{N}(0, \varsigma)\right)
$$

omitting $\xi=0=y_{0}$ so that $\Sigma^{2 N-1} \subset \mathbb{C}^{N}, S^{2 N-1} \subset \mathbb{C}^{N}$ to simplify the notation. By a Mayer-Vietoris $\operatorname{argument} \operatorname{deg} y=\operatorname{deg} Y$; for more details see [8, §2]. Finally define maps $\Phi, \Psi: \mathbb{C}^{N} \rightarrow \mathbb{C}^{N}$ by

$$
\begin{aligned}
& \Phi\left(\varsigma_{1}, \ldots, \varsigma_{N}\right)=\left(\varsigma_{1}^{m_{1}}, \varsigma_{2}^{m_{2}}, \ldots, \varsigma_{N}^{m_{N}}\right), \quad m_{j}:=N ! / j, \\
& \Psi\left(\varsigma_{1}, \ldots, \varsigma_{N}\right)=\left(\varsigma_{1}, \varsigma_{2}^{2}, \ldots, \varsigma_{N}^{N}\right),
\end{aligned}
$$

and

$$
Z:=\Phi \circ Y \circ \Psi: \Sigma^{2 N-1} \rightarrow S^{2 N-1} \text {. }
$$

A simple calculation shows that (4.1) implies the following equivariance property for $Z$ :

$$
Z(\alpha \cdot \varsigma)=\alpha^{2(N !)} Z(\varsigma)
$$

for all $\varsigma \in \Sigma^{2 N-1}$ and $\alpha \in \mathbb{C}$ with $|\alpha|=1$.

Invoking a theorem of Hopf and Rueff [26], this $S^{1}$-equivariance implies $Z$ is essential (this is the generalized Borsuk-Ulam theorem mentioned in the introduction). Hence $Y$ is essential, $\operatorname{deg} y=\operatorname{deg} Y \neq 0$. Thus $y=y^{\theta=1}$ and consequently $y^{\theta=0}$, our original $y$-map, are all essential. This completes the proof of Theorems 4.2 and 4.3 in case $i(U)=2 N+1$ is odd.

PROOF OF THEOREMS 4.2 AND 4.3 (FOR $i(U)=2 N$ ). In this case the spectrum of $T=d \phi_{t}(U)$ is given by $\lambda_{0}, \lambda_{1}, \ldots$ with $\lambda_{2 N-1}>1 \geq \lambda_{2 N}$ (recall that $\lambda_{2 N-1}$ and $\lambda_{2 N}$ are either complex conjugate or both real, and the first case is ruled 
out by our assumption that $i(U)$ is even). The strong unstable manifold $W^{\text {su }}$ is now $2 N$-dimensional and contains the fast unstable manifold $W^{\text {fu }}$ belonging to the eigenvalues $\lambda_{0}, \lambda_{1}, \ldots, \lambda_{2 N-2}\left(\mathrm{cf}\right.$. [7] for $\left.W^{\mathrm{fu}}\right)$.

Let $\Sigma^{2 N-1}$ denote a small sphere in $W^{\text {su }}$, as usual, and let $\Sigma^{2 N-2}$ denote its intersection with $W^{\text {fu }}$. Topologically $\Sigma^{2 N-2}$ is an equator in $\Sigma^{2 N-1}$.

On $\Sigma^{2 N-2}$ we define $t_{n}, \tau_{n}, \sigma, \theta_{n}$ as before (using $z(u-U) \leq 2 N-2$ on $W^{\text {fu }}$ [7]), and $\left(y_{0}, y_{1}, \ldots, y_{N-1}\right): \Sigma^{2 N-2} \rightarrow S^{2 N-2} \subset \mathbb{R} \times \mathbb{C}^{N-1}$ is essential. To define a $y$-map

$$
y: \Sigma^{2 N-1} \rightarrow S^{2 N-1} \subset \mathbb{R} \times \mathbb{C}^{N-1} \times \mathbb{R},
$$

$y=\left(y_{0}, y_{1}, \ldots, y_{N-1}, y_{N}\right)$, we need only one additional component $y_{N}: \Sigma^{2 N-1} \rightarrow \mathbb{R}$ such that $y$ becomes essential. From $\Sigma\left|y_{n}\right|^{2}=1$ we obtain

$$
\left|y_{N}\right|^{2}=\tau_{N-1}+\frac{1}{2}=\tau_{N-1}-\tau_{N}
$$

and we only need a sign for $y_{N}$. Since $\Sigma^{2 N-2}$ divides $\Sigma^{2 N-1}$ into an upper and a lower hemisphere we can define $y_{N}$ such that $y_{N} \geq 0$ (resp. $y_{N} \leq 0$ ) on the upper (resp. lower) hemisphere. Note that $y_{N}$ is now a continuous function on $\Sigma^{2 N-1}$, since $y_{N}$ vanishes on the equator $\Sigma^{2 N-2}$ (recall that $\tau_{N}=\tau_{N-1}=-\frac{1}{2}$ on $\left.\Sigma^{2 N-2}\right)$. Because $y$, thus defined, respects the partitioning of $\Sigma^{2 N-1}$ and $S^{2 N-1}$ into hemisphere, and because $y$ is essential on the equators $\Sigma^{2 N-2}$ and $S^{2 N-2}$, Mayer-Vietoris again implies that $y$ itself is essential as in [8, §2]. This completes the proof of Theorems 4.2 and 4.3, finally.

5. Appendix on the zero number. In this appendix we prove, assuming analyticity, that $z(u(t, \cdot))$ drops whenever $x \mapsto u(t, x)$ has a multiple zero. Recall that for any continuous function $\phi(x)\left(x \in S^{1}\right)$ the zero number $z(\phi)$ is defined to be the largest integer $n \leq \infty$ such that there exist $0 \leq x_{n+1}=x_{0}<x_{1}<\cdots<$ $x_{n}<2 \pi$ for which

$$
\phi\left(x_{i}\right) \cdot \phi\left(x_{i+1}\right)<0 \quad(i=0,1, \ldots, n) .
$$

If $\phi \in C^{1}\left(S^{1}\right)$ has only simple zeros, then $z(\phi)$ is simply the number of zeros of $u$.

Let $a, b, c$ be real analytic functions of $(t, x) \in S^{1} \times[0, \tau]$ for some $\tau>0, a>0$, and let $u(x, t)$ be a classical solution of the linear parabolic equation

$$
u_{t}=a(t, x) u_{x x}+b(t, x) u_{x}+c(t, x) u \quad\left(x \in S^{1}, 0 \leq t \leq \tau\right) .
$$

Then for $0<t \leq \tau, u$ is a real analytic function in $(x, t)$, cf. [29]. It is known under much weaker conditions that $z(u(t, \cdot))$ is a nonincreasing function of $t$.

Our assumption that all coefficients $a, b$ and $c$ are real analytic allows us to characterize those moments in time when $z(u(t, \cdot))$ drops.

THEOREM 5.1. If $x \mapsto u\left(t_{0}, x\right)$ has a multiple zero at $t_{0} \in(0, \tau)$, then $z(u(t, \cdot))$ drops at $t=t_{0}$.

We postpone the proof of this result, which will be based on a careful study of the zero set of $u(t, x)$ near a point where $u\left(t_{0}, \cdot\right)$ has a multiple zero. Near such a point we shall be able to compute the relevant part of the Taylor series to apply a classical method: the Newton polygon described e.g. in [5, pp. 494$518 ; 10, \S 2.8]$. The exhaustive treatment in [5] includes a very readable account of Newton's original work! The method implies that the local zero set of $u(t, x)$ 

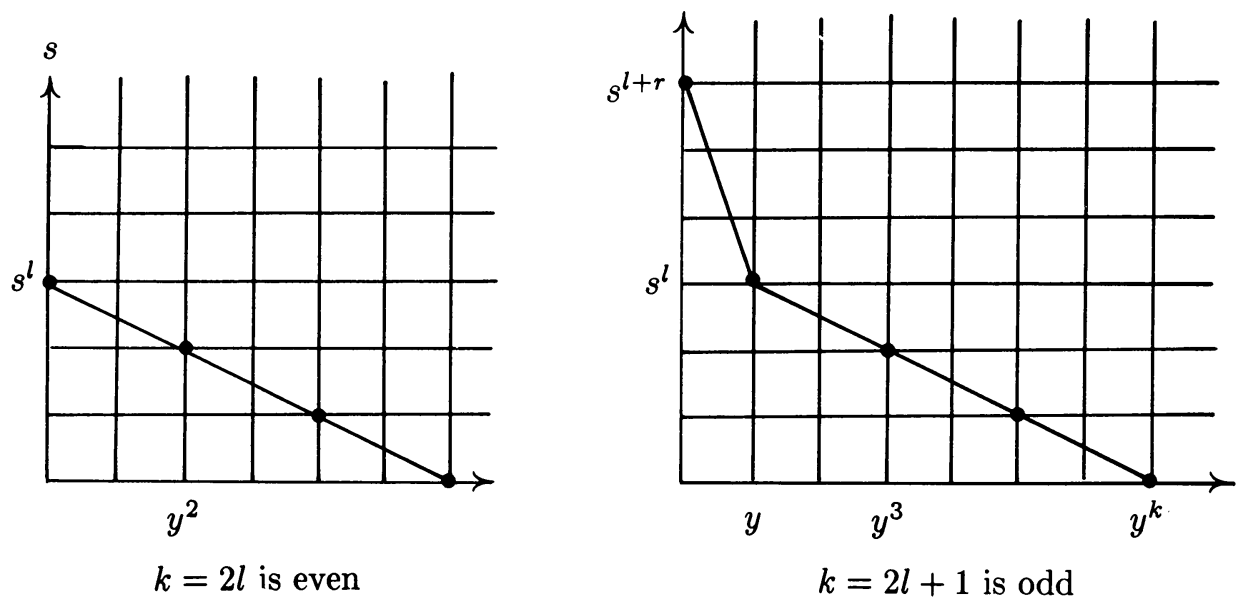

FIGURE 5.1. The Newton polygon of $u\left(t_{0}+s, x_{0}+y\right)$

consists of certain analytic curves. The way these curves are shaped will then imply the theorem.

From here on let $\left(t_{0}, x_{0}\right)$ be such that $u\left(t_{0}, \cdot\right)$ has a multiple zero at $x_{0}$. Write $x=x_{0}+y, t=t_{0}+s$, and assume that for fixed $t_{0}$

$$
u\left(t_{0}, x_{0}+y\right)=A \cdot y^{k}+O\left(y^{k+1}\right),
$$

where $A \neq 0, k \geq 2$, and $O\left(y^{k+1}\right)$ denotes an analytic function of $y$ divisible by $y^{k+1}$. We calculate the Taylor series of $(s, y) \mapsto u\left(t_{0}+s, x_{0}+y\right)$.

LEMMA 5.2. If $2 m \leq k$, then

$$
\partial_{t}^{m} u\left(t_{0}, x_{0}+y\right)=A a_{0}^{m} k ! \frac{y^{k-2 m}}{(k-2 m) !}+O\left(y^{k-2 m+1}\right),
$$

where $a_{0}=a\left(t_{0}, x_{0}\right)$.

PROOF (BY INDUCTION ON $m$ ). For simplicity we assume that $A=1$. For $m=0$ the result is trivially true. Let the lemma be proved for $m \leq m_{0}$. Then

$$
\begin{aligned}
\partial_{t}^{m_{0}+1} u & =\partial_{t}^{m_{0}}\left\{a u_{x x}+b u_{x}+c u\right\} \\
& =\sum_{m+l=m_{0}}\left(\begin{array}{c}
m_{0} \\
m
\end{array}\right)\left\{\partial_{t}^{m} a\left(\partial_{t}^{l} u\right)_{x x}+\partial_{t}^{m} b\left(\partial_{t}^{l} u\right)_{x}+\partial_{t}^{m} c \cdot \partial_{t}^{l} u\right\},
\end{aligned}
$$

by Leibniz's rule.

The induction hypothesis implies that there is only one term which is not divisible by $y^{k-2 m_{0}-1}$, namely

$$
\begin{aligned}
\partial_{t}^{m_{0}+1} u\left(t_{0}, x_{0}+y\right) & =a\left(t_{0}, x_{0}\right)\left(\partial_{t}^{m_{0}} u\right)_{x x}+O\left(y^{k-2 m_{0}-1}\right) \\
& =a_{0}^{m_{0}+1} \frac{k !}{\left(k-2 m_{0}-2\right) !} y^{k-2 m_{0}-2}+O\left(y^{k-2 m_{0}-1}\right),
\end{aligned}
$$

which proves the lemma. 
This lemma enables us to compute the Newton polygon of the Taylor series of $u\left(t_{0}+s, x_{0}+y\right)$ at $(0,0)$. In fact, let

$$
u\left(t_{0}+s, x_{0}+y\right)=\sum_{m, n} u_{m n} y^{m} s^{n}
$$

then $u_{m n}=0$ if $m+2 n<k$. The Newton polygon consists of the convex hull of $\left\{(m, n): u_{m n} \neq 0\right\}$ from below. If $k=2 l$ is even, then the Newton polygon is given by the straight line through $(k, 0)$ and $(0, l)$. Otherwise, if $k=2 l+1$, then the Newton polygon consists of two line segments: One from $(k, 0)$ to $(1, l)$, and one from $(1, l)$ to $(0, l+r)$ for some $1 \leq r \leq \infty$.

PROOF OF THEOREM 5.1. To exhaust the zero set of $u$ near $\left(t_{0}, x_{0}\right)$ the Newton polygon method first suggests to consider the analytic function

$$
\phi(\sigma, y)=y^{-k} \cdot u\left(t_{0}+y^{2} \sigma, x_{0}+y\right)=\sum_{m, n} u_{m n} y^{m+2 n-k} \sigma^{n}
$$

for small $|y|$. By the implicit function theorem, we obtain analytic solution curves

$$
\sigma=\sigma_{j}(y) \Leftrightarrow s=y^{2} \sigma_{j}(y), \quad 1 \leq j \leq 2 l
$$

if

$$
U(\sigma):=\Phi(\sigma, 0)=\sum_{m+2 n=k} u_{m n} \sigma^{n}
$$

has only simple zeros $\sigma=\sigma_{j}(0)$ (here $k=2 l$ or $2 l+1$ ). We employ Lemma 5.2 to calculate the polynomial $U(\sigma)$ explicitly as

$$
U(\sigma)=k ! \sum_{2 n \leq k} \frac{1}{n !(k-2 n) !}\left(a_{0} \sigma\right)^{n} .
$$

En passant we mention that the relevant part $\tilde{u}$ of $u$,

$$
\tilde{u}(s, y):=y^{k} U\left(\frac{s}{y^{2}}\right)=\sum_{m+2 n=k} u_{m n} y^{m} s^{n},
$$

satisfies the standard heat equation

$$
\tilde{u}_{s}=a_{0} \tilde{u}_{y y} .
$$

Note that $U(\sigma)$ relates to the $k$ th Hermite polynomial $H_{k}(z)$ (in the notation of [1]) as follows:

$$
\begin{aligned}
U(\sigma) & =k !(\sqrt{-\sigma})^{k} \sum_{2 n \leq k} \frac{(-1)^{n}}{n !(k-2 n) !}\left(\frac{1}{\sqrt{-\sigma}}\right)^{k-2 n} \\
& =k !(\sqrt{-\sigma})^{k} H_{k}\left(\frac{1}{2 \sqrt{-\sigma}}\right)
\end{aligned}
$$

choosing a sign for $\sqrt{-\sigma}$, consistently. In fact, $H_{k}(z)$ has only real simple zeros. Hence all zeros of $U(\sigma)$ are indeed simple, and negative. For $u(t, x)=u\left(t_{0}+s, x_{0}+y\right)$ we thus obtain $l$ parabolic curves of zeros

$$
s=y^{2} \sigma_{j}(y), \quad 1 \leq j \leq l,
$$

whose branches point backwards in time, i.e. they are contained in $\left\{t \leq t_{0}\right\}$. 
In case $k=2 l$ is even, these curves exhaust the zero set of $u$ near $\left(t_{0}, x_{0}\right)$, cf. [5, 10]. In case $k=2 l+1$ and $r=\infty$ there is exactly one additional curve of zeros given by $y=y(s)=0$. Finally, we have to consider the case $k=2 l+1,1 \leq r<\infty$. In that case we solve

$$
\begin{aligned}
\phi(s, \eta) & :=s^{-(l+r)} u\left(t_{0}+s, x_{0}+s^{r} \eta\right) \\
& =\sum_{m, n} u_{m n} \eta^{m} s^{r m+n-(l+r)}
\end{aligned}
$$

for small $|s|$. Again, we obtain an analytic solution curve

$$
\eta=\eta(s) \Leftrightarrow y=s^{r} \eta(s)
$$

starting at the simple zero $\eta=\eta(0) \neq 0$ of $\eta \rightarrow \phi(0, \eta)=u_{0, l+r}+u_{1, l} \eta$. As before, the Newton polygon method implies that the curves (4.2), (4.3) exhaust the zero set of $u(t, x)$ near $\left(t_{0}, x_{0}\right)$.

In any case, $u(t, \cdot)$ has near $x_{0}$

$$
\begin{aligned}
\text { at most one zero, } & \text { if } t>t_{0}, \\
\text { exactly } k \text { zeros, } & \text { if } t<t_{0},
\end{aligned}
$$

provided $\left|t-t_{0}\right|$ is small. All these zeros are simple by the implicit function theorem argument above. Hence at least $k-1$ zeros of $u(t, \cdot)$ disappear as $t$ crosses $t_{0}$. Since we had assumed that $x_{0}$ was a multiple zero we have $k \geq 2$, so that Theorem 5.1 is true.

We conclude this appendix with the following corollary of Theorem 5.1:

COROLLARY 5.3. Let $u, v$ be classical solutions of

$$
u_{t}=u_{x x}+f\left(x, t, u, u_{x}\right) \quad x \in S^{1}, 0<t \leq \tau,
$$

where $f: S^{1} \times[0, \tau] \times \mathbb{R}^{2} \rightarrow \mathbb{R}$ is real analytic. Then, $z(u(t, \cdot)-v(t, \cdot))$ is finite and nonincreasing in $t$ for any $t \in(0, \tau]$. Furthermore, if $z(u(t, \cdot)-v(t, \cdot))$ is constant near $t=t_{0}$, then $u\left(t_{0}, \cdot\right)-v\left(t_{0}, \cdot\right)$ has only simple zeros.

PROOF. The solutions $u$ and $v$ are analytic on $(0, \tau] \times S^{1}$ [29]. Now consider $w(t, x)=u(t, x)-v(t, x)$. This function satisfies

$$
w_{t}=w_{x x}+a(t, x) w_{x}+b(t, x) w, \quad x \in S^{1}, 0<t \leq \tau,
$$

where

$$
\begin{aligned}
& a(x, t)=\int_{0}^{1} f_{p}\left(x, t, u(\theta, t, x), u_{x}(\theta, t, x)\right) d \theta \\
& b(x, t)=\int_{0}^{1} f_{u}\left(x, t, u(\theta, t, x), u_{x}(\theta, t, x)\right) d \theta
\end{aligned}
$$

and

$$
u(\theta, t, x)=\theta u(t, x)+(1-\theta) v(t, x) .
$$

Clearly $a$ and $b$ are analytic on $(0, \tau] \times S^{1}$ so that we can apply Theorem 5.1 to $w$ and thereby complete the proof.

NOTE ADDED IN PROOF. After this paper was written we were informed that both $\mathrm{H}$. Matano and P. Massat have independently considered the very same problem which we had considered. There is some overlap between their results and ours. 
In addition, together with J. Mallet-Paret, the second author (B.F.) has been able to resolve the problem mentioned in Remark 3.2, concerning the uniqueness of the rotating wave in an omega-limit set. These results will be published in a forthcoming paper on Poincaré Bendixon theory for scalar reaction diffusion equations. Finally, the first author (S.B.A.) has found that Theorem 5.1 also holds for nonanalytic coefficients. Thus the results of this paper are true if the nonlinearity $f$ has two continuous derivatives. The proof of Theorem 5.1 in the nonsmooth case will appear in the Journal für die reine und angewandte Mathematik under the title The zeroset of a solution of a parabolic equations.

\section{REFERENCES}

1. M. Abramowitz and I. A. Stegun (Editors), Handbook of mathematical functions, Dover, New York, 1965.

2. H. Amann, Global existence for semilinear parabolic systems, J. Reine Angew. Math. 360 (1985), 47-83.

3. S. Angenent, The Morse-Smale property for a semilinear parabolic equation, J. Differential Equations 62 (1986), 427-442.

4. B. Aulbach, Continuous and discrete dynamics near manifolds of equilibria, Lecture Notes in Math., vol. 1058, Springer-Verlag, Berlin, 1984.

5. E. Brieskorn and H. Knörrer, Ebene algebraische Kurven, Birkhäuser, Basel, 1981.

6. P. Brunovský and B. Fiedler, Simplicity of zeros in scalar parabolic equations, J. Differential Equations 62 (1986), 237-241.

7. _ Zero numbers on invariant manifolds in scalar reaction diffusion equations, Nonlinear Anal. 10 (1986), 179-194.

8. __ Connecting orbits in scalar reaction diffusion equations, Dynamics Reported (U. Kirchgraber, ed.) (to appear).

9. K.-S. Cheng, Decay rate of periodic solutions for a conservation law, J. Differential Equations 42 (1981), 390-399.

10. S.-N. Chow and J. K. Hale, Methods of bifurcation theory, Springer-Verlag, New York, 1982.

11. C. C. Conley and J. Smoller, Topological techniques in reaction diffusion equations, Biological Growth and Spread (Proc. Heidelberg 1979) (W. Jäger, H. Rost and P. Tautu, eds.), Lecture Notes in Biomath., vol. 38, Springer-Verlag, Heidelberg, pp. 473-483.

12. I. P. Cornfeld, S. V. Fomin and Y. G. Sinai, Ergodic theory, Springer-Verlag, New York, 1982.

13. C. M. Dafermos, Applications of the invariance principle for compact processes. II: Asymptotic behaviour of solutions of a hyperbolic conservation law, J. Differential Equations 11 (1972), 416-424.

14. A. Dold, Lectures on algebraic topology, Springer-Verlag, Heidelberg, 1972.

15. J. Dugundji, Topology, Allyn \& Bacon, Boston, Mass.,1966.

16. J. J. Duistermaat, Stable manifolds, preprint no. 40, Math. Inst., Utrecht, 1976.

17. J. Guckenheimer and P. J. Holmes, Nonlinear oscillations, dynamical systems, and bifurcations of vector fields, Springer-Verlag, New York, 1983.

18. V. Guillemin and A. Pollack, Differential topology, Prentice-Hall, Englewood Cliffs, N.J., 1974.

19. J. K. Hale, Infinite dimensional dynamical systems, Geometric Dynamics (Proc. Rio de Janeiro 1981) (J. Palis, Jr., ed.), Lecture Notes in Math., vol. 1007, Springer-Verlag, New York, pp. 379-400.

20. D. Henry, Geometric theory of semilinear parabolic equations, Lecture Notes in Math., vol. 840, Springer-Verlag, New York, 1981.

21. __ Some infinite dimensional Morse-Smale systems defined by parabolic differential equations,

J. Differential Equations 59 (1985), 165-205.

22. M. W. Hirsch, Differential topology, Springer-Verlag, New York, 1976.

23. __ Differential equations and convergence almost everywhere in strongly monotone semiflows, Nonlinear Partial Differential Equations (J. Smoller, ed.), Amer. Math. Soc., Providence, R. I., 1983, pp. 267-285. 
24. __ Systems of differential equations that are competitive or cooperative. II: Convergence almost everywhere, SIAM J. Math. Anal. 16 (1985), 423-439.

25. M. W. Hirsch, C. C. Pugh and M. Shub, Invariant manifolds, Lecture Notes in Math., vol. 583, Springer-Verlag, Berlin, 1977.

26. H. Hopf and M. Rueff, Über faserungstreue Abbildungen der Sphären, Comment. Math. Helv. 11 (1938), 49-61.

27. T. Kato, Perturbation theory for linear operators, Springer-Verlag, New York, 1980.

28. J. Kevorkian and J. D. Cole, Perturbation methods in applied mathematics, Springer-Verlag, New York, 1981.

29. G. Komatsu, Analyticity up to the boundary of solutions of nonlinear parabolic equations, Comm. Pure Appl. Math. 32 (1979), 669-720.

30. P. D. Lax, Hyperbolic systems of conservation laws. II, Comm. Pure Appl. Math. 10 (1957), 537-556.

31. J. Mallet-Paret, Morse decompositions and global continuation of periodic solutions for singularly perturbed delay equations, Systems of Nonlinear Partial Differential Equations (J. M. Ball, ed.), Reidel, Dordrecht, 1983, pp. 351-366.

32. __ Morse decompositions for delay-differential equations, preprint.

33. J. Mallet-Paret and B. Fiedler, Connections of Morse sets for delay-differential equations, in preparation.

34. W. S. Massey, Singular homology theory, Springer-Verlag, New York, 1980.

35. H. Matano, Convergence of solutions of one-dimensional parabolic equations, J. Math. Kyoto Univ. 18 (1978), 221-227.

36. _ _ Nonincrease of the lap-number of a solution for a one-dimensional semi-linear parabolic equation, J. Fac. Sci. Univ. Tokyo Sect. IA 29 (1982), 401-441.

37. _ Existence of nontrivial unstable sets for equilibriums of strongly order-preserving systems, J. Fac. Sci. Univ. Tokyo Sect. IA 30 (1984), 645-673.

38. , personal communication, 1986.

39. E. F. Mishchenko and N. K. Rozov, Differential equations with small parameters and relaxation oscillations, Plenum Press, New York, 1980.

40. K. Nickel, Gestaltaussagen über Lösungen parabolischer Differentialgleichungen, J. Reine Angew. Math. 211 (1962), 78-94.

41. O. A. Oleinik and S. N. Kruzhkov, Quasilinear second order parabolic equations with many independent variables, Russian Math. Surveys 16 (1961), 105-146.

42. K. Petersen, Ergodic theory, Cambridge University Press, Cambridge, 1983.

43. J. Smoller, Shock waves and reaction diffusion equations, Springer-Verlag, New York, 1983.

\footnotetext{
RiJKUniversiteit te Leiden, SUbF. Wiskunde EN INFORMATICA, 2300 RA LEIDEN, THE NETHERLANDS

SONDERFORSCHUNGSBEREICH 123, UNIVERSITÄT HEIDELBERG, D-6900 HeIDELBERG, WEST GERMANY (Current address of B. Fiedler)
}

Current address (S. B. Angenent): Department of Mathematics University of Wisconsin, Madison, Wisconsin 53706 